\title{
Quad/triangle Subdivision, Nonhomogeneous Refinement Equation and Polynomial Reproduction
}

\author{
Qingtang Jianga,*, Baobin Li ${ }^{\mathrm{b},}$ \\ ${ }^{a}$ Department of Mathematics and Computer Science, University of Missouri-St. Louis, \\ St. Louis, MO 63121, U.S.A. \\ ${ }^{b}$ School of Information Science and Engineering, Graduate University of Chinese \\ Academy of Sciences, Beijing, 100190, P.R.China.
}

\begin{abstract}
The quad/triangular subdivision, whose control net and refined meshes consist of both quads and triangles, provides better visual quality of subdivision surfaces. While some theoretical results such as polynomial reproduction and smoothness analysis of quad/triangle schemes have been obtained in the literature, some issues such as the basis functions at quad/triangle vertices and design of interpolatory quad/triangle schemes need further study. In our study of quad/triangle schemes, we observe that a quad/triangle subdivision scheme can be derived from a nonhomogeneous refinement equation. Hence, the basis functions at quad/triangle vertices are shifts of the refinable function associated with a nonhomogeneous refinement equation. In this paper a quad/triangle subdivision surface is expressed analytically as the linear combination of these basis functions and the polynomial reproduction of matrix-valued quad/triangle schemes is studied. The result on polynomial reproduction achieved here is critical for the smoothness analysis and construction of matrix-valued quad/triangle schemes. Several new schemes are also constructed.
\end{abstract}

Keywords: Quad/triangle subdivision, nonhomogeneous refinement equation, basis function, subdivision limiting surface, polynomial reproduction, interpolatory scheme

\footnotetext{
*Corresponding author.Tel.: +01 314-516-6358; fax: +01 314-516-5400.

Email addresses: jiangq@umsl.edu (Qingtang Jiang), libb@gucas.ac.cn (Baobin $\mathrm{Li})$
} 


\section{Introduction}

The subdivision process is carried out iteratively from an initial polyhedron, called control net or control mesh, to generate finer and finer meshes. The control mesh and its finer meshes produced by a conventional subdivision scheme consist of either quadrilateral (quads) or triangles, but not both. More precisely, a quad subdivision scheme starts with a control net consisting of quads, and generated finer and finer meshes of quads, while a triangle subdivision scheme generates triangular meshes from an initial triangular mesh. For either the quad or triangle subdivision, the dyadic (or 1-to-4 split) refinement is the most commonly used refinement. During each iteration step of a dyadic subdivision, one quad (triangle resp.) is split into four quads (triangles resp.). During this process, new vertices (also called "odd" vertices) are inserted among old vertices (also called "even" vertices) on the coarser mesh (i.e. the mesh before the next iteration step is carried out) and the positions of old vertices may be changed. The exact positions of the "odd" vertices (and possibly "even" vertices) in the finer mesh are given by the local averaging rule. If the positions of "even" vertices are not changed during the subdivision process, then the subdivision scheme is called an interpolatory scheme. Otherwise, it is called an approximation scheme.

The local averaging rule (for regular vertices) is associated with a certain refinement equation

$$
\phi(\mathbf{x})=\sum_{\mathbf{k} \in \mathbf{Z}^{2}} p_{\mathbf{k}} \phi(2 \mathbf{x}-\mathbf{k}), \quad \mathbf{x} \in \mathbb{R}^{2}
$$

with a (finite) sequence $\left\{p_{\mathbf{k}}\right\}$ called the refinement mask or subdivision mask, and a compactly supported function $\phi(\mathbf{x})$ called the refinable function. For a (regular) control net with vertices $v_{\mathbf{k}}^{0}$, the refinement equation (1.1) yields the local averaging rule:

$$
v_{\mathbf{k}}^{\ell+1}=\sum_{\mathbf{n} \in \mathbf{Z}^{2}} v_{\mathbf{n}}^{\ell} p_{\mathbf{k}-2 \mathbf{n}}, \quad \mathbf{k} \in \mathbb{Z}^{2}, \ell=0,1, \cdots,
$$

where $v_{\mathbf{k}}^{\ell+1}$ are the vertices of the refined mesh obtained after $\ell+1$ steps of subdivision iterations. The local averaging rule (1.2) is sometimes described and represented in the plane with a set of subdivision templates (stencils). The finer and finer polyhedra with vertices $v_{\mathbf{k}}^{\ell}$ give an approximation to the limiting surface given by $\sum_{\mathbf{k}} v_{\mathbf{k}}^{0} \phi(\mathbf{x}-\mathbf{k})$. In particular, if the initial net is 

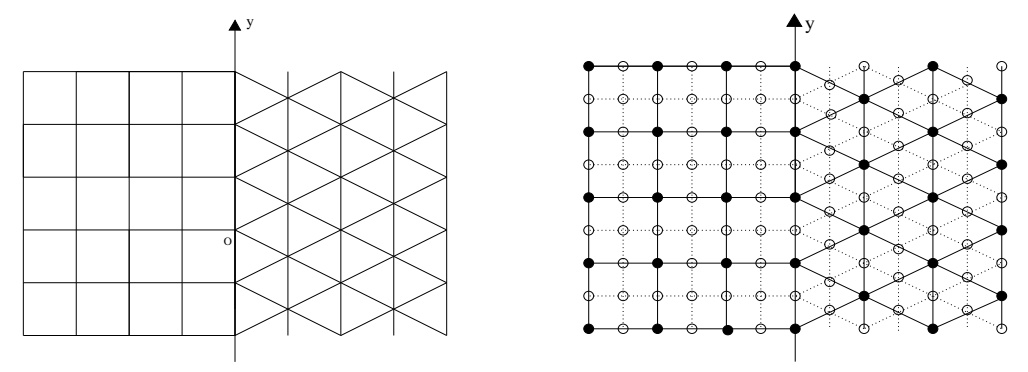

Figure 1: Quad/triangle mesh (left) and refined quad/triangle mesh (right)

$\delta_{\mathbf{k}}$, the kronecker-delta sequence, then the limit surface is $\phi(\mathbf{x}-\mathbf{k})$. Thus $\phi(\mathbf{x}-\mathbf{k})$ is also called the basis function at $\mathbf{k}$.

Catmull-Clark's scheme [2] and Loop's scheme [11] are the two most commonly used schemes. The former is for the quad mesh and the latter for the triangle mesh. In CAD modeling, the designers often want to model certain regions with quad meshes and others with triangle meshes to get better visual quality of subdivision surfaces. Thus, it is desirable to have surfaces that have a hybrid quad/triangle mesh structure (see e.g. [16]). The quad/triangle subdivision was introduced for this purpose (see [12, 16, 15], and refer to [13] for its advantages and to $[9,10]$ for the polynomial reproduction and smoothness analysis). A subdivision algorithm for a regular quad/triangle vertex, a vertex surrounded by 2 adjacent quads and 3 adjacent triangles, can be represented in the parametric plane as the origin of the mesh in the left picture of Fig.1, where all vertices on the $y$-axis are called quad/triangle vertices. During the quad/triangle subdivision, "odd" vertices (denoted as $\circ$ in the right picture of Fig.1) are inserted among the "even" vertices (denoted as $\bullet$ ), and then, they are connected appropriately such that each quad and triangle in the coarser mesh are split into 4 quads and 4 triangles in the finer mesh, see the right picture of Fig.1. The exact positions of the "odd" and "even" vertices in the finer mesh are given by the local averaging rule.

For a conventional quad or triangle subdivision, the basis function at a regular vertex is an integer-shift of the refinable function $\phi$. For the quad/triangle subdivision, we have the basic question: what is the basis function at a quad/triangle vertex? Whether it is also related to a refinable function, and whether a quad/triangle scheme can be derived a refinement equation similar to (1.1)? If yes, whether the subdivision surface is also given as the series of the basis functions with the control vertices as the coefficients? 
In our study of quad/triagle schemes, we realize that the above issues are associated with the nonhomogeneous refinement equation of the form

$$
\boldsymbol{\phi}(\mathbf{x})=\sum_{\mathbf{k} \in \mathbf{Z}^{2}} A_{\mathbf{k}} \boldsymbol{\phi}(2 \mathbf{x}-\mathbf{k})+N_{0}(\mathbf{x}), \quad \mathbf{x} \in \mathbb{R}^{2},
$$

where $\boldsymbol{\phi}=\left[\phi_{0}, \phi_{1}, \cdots, \phi_{r-1}\right]^{T}$ for some $r$ is called refinable function vector, and $N_{0}(\mathbf{x})$ is called the nonhomogeneous term. In our paper [8], we show that a quad/triangle subdivision scheme with either scalar-valued templates or matrix-valued templates can be derived from a nonhomogeneous refinement equation. Thus, the basis functions at quad/triangle vertices are the integer shifts of the refinable functions associated with nonhomogeneous refinement equations. In this paper, we obtain that the limiting surfaces can be expressed as the linear combination of the shifts of the refinable functions. For the purpose of constructing interpolatory quad/triangle schemes, in this paper we also study polynomial reproduction of matrix-valued quad/triangle schemes. The result on polynomial reproduction obtained in this paper leads to the construction of matrix-valued $C^{2}$ interpolatory quad/triagle schemes. The analytical expression of a quad/triangle subdivision limiting surface is obtained in $\S 2$, and the polynomial reproduction of quad/triangle schemes with both scalar-valued and matrix-valued schemes is presented in a uniform way in $\S 3$. With the conditions for polynomial reproduction obtained in $\S 3$, we construct a few new quad/triangle schemes in $\S 4$.

In this paper we consider subdivision schemes for regular vertices only. Thus, in the following, a vertex means a regular vertex, namely, it has valence 4 if it is a quad vertex; it has valence 6 if it is a triangle vertex; and it is surrounded by 2 adjacent quads and 3 adjacent triangles if it is a quad/triangle vertex. Throughout this paper, we use $\Gamma_{1}$ and $\Gamma_{2}$ to denote the subsets of $\mathbb{Z}^{2}: \Gamma_{1}:=\left\{\mathbf{n}=\left(n_{1}, n_{2}\right): n_{1} \leq-2, n_{1}, n_{2} \in \mathbb{Z}\right\}, \Gamma_{2}:=\left\{\mathbf{m}=\left(m_{1}, m_{2}\right):\right.$ $\left.m_{1} \geq 2, m_{1}, m_{2} \in \mathbb{Z}\right\}$. For $\mathbf{m}=\left(m_{1}, m_{2}\right) \in \mathbb{Z}^{2}$, denote

$$
\widetilde{\mathbf{m}}:= \begin{cases}\mathbf{m}, & \text { if } m_{1} \text { is even, } \\ \left(m_{1}, m_{2}-\frac{1}{2}\right), & \text { if } m_{1} \text { is odd. }\end{cases}
$$

\section{Nonhomogeneous refinement equation, quad/triangle scheme and limiting surface}

2.1. Nonhomogeneous refinement equation and quad/triangle scheme

Recently in their study of matrix-valued templates for surface design, the authors in [3] introduce a natural definition of matrix-valued interpolatory 
scheme which results in interpolatory schemes with small supports. In this subsection we show that a scalar or matrix-valued quad/triangle scheme can be derived from a nonhomogeneous refinement equation.
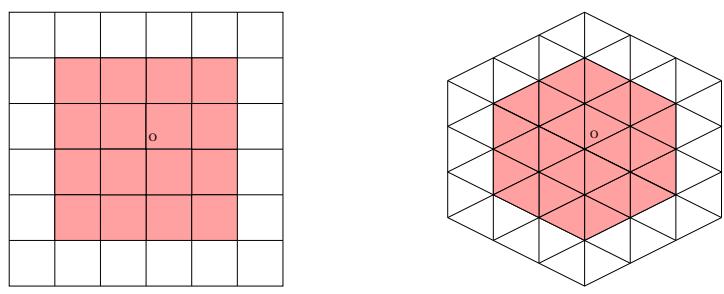

Figure 2: Quad grid (left) and triangle grid (right)

Let $S(\mathbf{x})=\left[S_{0}(\mathbf{x}), S_{1}(\mathbf{x}), \cdots, S_{r-1}(\mathbf{x})\right]^{T}$ and $T(\mathbf{x})=\left[T_{0}(\mathbf{x}), T_{1}(\mathbf{x}), \cdots\right.$, $\left.T_{r-1}(\mathbf{x})\right]^{T}$ be the basis function vectors for the quad grid and triangle grid respectively in a matrix-valued quad/triangle scheme. More precisely, $S(\mathbf{x})$ is refinable along lattice $\mathbb{Z}^{2}$ with the grid shown on the left of Fig.2 and $T(\mathbf{x})$ is refinable along lattice $\{(2 j, k): j, k \in \mathbb{Z}\} \cup\left\{\left(2 j+1, k+\frac{1}{2}\right): j, k \in \mathbb{Z}\right\}$ with the grid shown on the right of Fig.2:

$$
\begin{aligned}
& S(\mathbf{x})=\sum_{\mathbf{k} \in \mathbf{Z}^{2}} Q_{\mathbf{k}} S(2 \mathbf{x}-\mathbf{k}), \mathbf{x} \in \mathbb{R}^{2}, \\
& T(\mathbf{x})=\sum_{\mathbf{k} \in \mathbf{Z}^{2}} P_{\mathbf{k}} T(2 \mathbf{x}-\widetilde{\mathbf{k}}), \mathbf{x} \in \mathbb{R}^{2}
\end{aligned}
$$

for some $r \times r$ constant matrices $Q_{\mathbf{k}}, P_{\mathbf{k}}$ with finitely many nonzeros, where for $\mathbf{k} \in \mathbb{Z}^{2}, \widetilde{\mathbf{k}}$ is defined in (1.4). Thus mask $\left\{Q_{\mathbf{k}}\right\}$ is used for quad vertices on the left but far from the $y$-axis of the quad/triangle grid (separated along the $y$-axis) shown in Fig.1, and $\left\{P_{\mathbf{k}}\right\}$ is used for triangle vertices on the right but far from the $y$-axis. For simplicity of presentation of the paper, we assume that each $S_{j}(\mathbf{x})$ is supported on a neighborhood of the origin consisting of 2-ring quads (the shadowed region on the left of Fig.2) and each $T_{j}(\mathbf{x})$ is supported on a neighborhood of the origin consisting of 2-ring triangles (the shadowed region on the right of Fig.2). We also assume that both $S(\mathbf{x})$ and $T(\mathbf{x})$ satisfy the partition unity property

$$
\mathbf{y}_{0} \sum_{\mathbf{k} \in \mathbf{Z}^{2}} S(\mathbf{x}-\mathbf{k})=1, \quad \mathbf{y}_{0} \sum_{\mathbf{k} \in \mathbf{Z}^{2}} T(\mathbf{x}-\widetilde{\mathbf{k}})=1, \mathbf{x} \in \mathbb{R}^{2},
$$

with

$$
\mathbf{y}_{0}=[1,0, \cdots, 0] .
$$


When the matrix-valued refinement equation (2.1) or (2.2) is applied to surface subdivisions, the local averaging rule for either the quad subdivision $\left(G_{\mathbf{k}}=Q_{\mathbf{k}}\right)$ or the triangle subdivision $\left(G_{\mathbf{k}}=P_{\mathbf{k}}\right)$ is given by

$$
\mathbf{v}_{\mathbf{k}}^{\ell+1}=\sum_{\mathbf{j}} \mathbf{v}_{\mathbf{j}}^{\ell} G_{\mathbf{k}-2 \mathbf{j}}, \quad \ell=0,1, \cdots,
$$

where

$$
\mathbf{v}_{\mathbf{k}}^{\ell}=:\left[v_{\mathbf{k}}^{\ell}, s_{\mathbf{k}, 1}^{\ell} \cdots, s_{\mathbf{k}, r-1}^{\ell}\right]
$$

are "row-vectors" with $r$ components of points $v_{\mathbf{k}}^{\ell}, s_{\mathbf{k}, i}^{\ell}, i=1, \cdots, r-1$, in $\mathbb{R}^{3}$. With $S(\mathbf{x}), T(\mathbf{x})$ satisfying $(2.3)$, as in [3,4], we may use the first components $v_{\mathbf{k}}^{\ell}$ of $\mathbf{v}_{\mathbf{k}}^{\ell}$ to denote the vertices of the subdivision meshes generated after the $\ell$ steps of iterations, with $v_{\mathbf{k}}^{0}$ being the initial vertices on the control net. The other components $s_{\mathbf{k}, 1}^{0}, \cdots, s_{\mathbf{k}, r-1}^{0}$ of $\mathbf{v}_{\mathbf{k}}^{0}$, can be used to control the surface geometric shape. In [3], the scheme is said to be interpolatory if $v_{2 \mathbf{k}}^{\ell+1}=v_{\mathbf{k}}^{\ell}$, namely, the vertices on the coarse mesh remain in the refined mesh. With this definition of interpolatory, $C^{2}$ interpolatory quad subdivision schemes with the matrix-valued templates having the same sizes as those of CatmullClark's scheme and $C^{2}$ interpolatory triangle subdivision schemes with the matrix-valued templates having the same sizes as those of Loop's scheme are constructed in [3]. For example, with templates in Figs. 3 and 4, where

$$
\begin{aligned}
& R=\left[\begin{array}{cc}
1, & -\frac{129}{64} \\
0, & -\frac{43}{128}
\end{array}\right], J=\left[\begin{array}{cc}
\frac{3}{8}, & 0 \\
-\frac{11}{128}, & \frac{17}{128}
\end{array}\right], K=\left[\begin{array}{cc}
\frac{1}{4}, & 0 \\
-\frac{1}{16}, & \frac{1}{16}
\end{array}\right], \\
& L=\left[\begin{array}{cc}
0, & \frac{99}{256} \\
0, & -\frac{33}{256}
\end{array}\right], M=\left[\begin{array}{cc}
\frac{1}{16}, & 0 \\
-\frac{5}{256}, & -\frac{1}{256}
\end{array}\right], N=\left[\begin{array}{cc}
0, & \frac{15}{128} \\
0, & -\frac{9}{256}
\end{array}\right],
\end{aligned}
$$

and

$$
\begin{aligned}
& P=\left[\begin{array}{cc}
1, & -\frac{435}{256} \\
0, & -\frac{91}{256}
\end{array}\right], B=\left[\begin{array}{cc}
\frac{3}{8}, & 0 \\
-\frac{47}{512}, & \frac{69}{512}
\end{array}\right], \\
& C=\left[\begin{array}{cc}
\frac{1}{8}, & 0 \\
-\frac{17}{512}, & -\frac{5}{512}
\end{array}\right], D=\left[\begin{array}{cc}
\frac{145}{512} \\
0, & -\frac{45}{512}
\end{array}\right],
\end{aligned}
$$

one has an interpolatory quad scheme and an interpolatory triangle scheme with the associated refinable function vectors in $C^{2}$, see [3].

Now let us return back to matrix-valued quad/triangle schemes. Consider

$$
\begin{gathered}
V_{0}:=\operatorname{span}_{L^{2}}\left\{\varphi_{j}(x, y-k), f_{j}(x, y-k), g_{j}(x, y-k), S_{j}(\mathbf{x}-\mathbf{n}),\right. \\
\left.T_{j}(\mathbf{x}-\widetilde{\mathbf{m}}): 0 \leq j \leq r-1, k \in \mathbb{Z}, \mathbf{n} \in \Gamma_{1}, \mathbf{m} \in \Gamma_{2}\right\},
\end{gathered}
$$



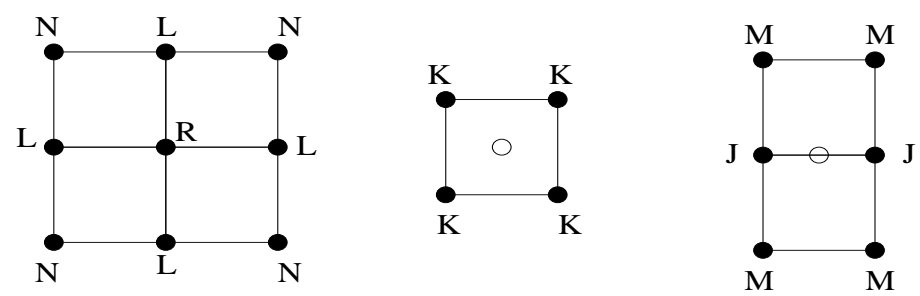

Figure 3: Matrix-valued templates of quad subdivision scheme for "even" vertices (left) and "odd" vertices (right)
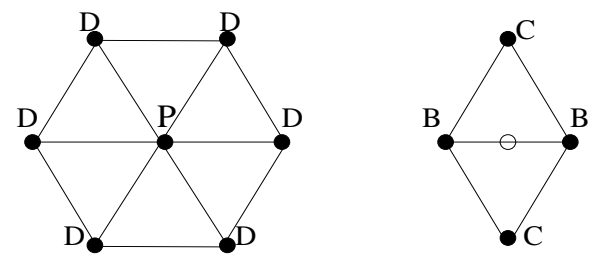

Figure 4: Matrix-valued templates of triangle subdivision scheme for "even" vertices (left) and "odd" vertices (right)

and $V_{\ell}:=\left\{F(\mathbf{x}): F\left(\frac{\mathbf{x}}{2^{\ell}}\right) \in V_{0}\right\}$, where $\varphi_{j}(\mathbf{x}), f_{j}(\mathbf{x})$ and $g_{j}(\mathbf{x})$ are compactly supported functions with $\operatorname{supp}\left(\varphi_{j}\right) \subset[-2,2] \times \mathbb{R}, \operatorname{supp}\left(f_{j}\right) \subset[-3,1] \times \mathbb{R}$, $\operatorname{supp}\left(g_{j}\right) \subset[-1,3] \times \mathbb{R}, 0 \leq j \leq r-1$. To assure that $\left\{V_{\ell}\right\}_{\ell}$ is nested, $\boldsymbol{\varphi}:=\left[\varphi_{0}, \varphi_{1}, \cdots, \varphi_{r-1}\right]^{T}, \mathbf{f}:=\left[f_{0}, f_{1}, \cdots, f_{r-1}\right]^{T}$ and $\mathbf{g}:=\left[g_{0}, g_{1}, \cdots, g_{r-1}\right]^{T}$ satisfy the following refinement relation:

$$
\begin{gathered}
\boldsymbol{\varphi}(\mathbf{x})=\sum_{k \in \mathbf{Z}} \mathbf{a}_{0, k} \boldsymbol{\varphi}(2 x, 2 y-k)+\sum_{k \in \mathbf{Z}} \mathbf{a}_{1, k} \mathbf{g}(2 x, 2 y-k)+ \\
\sum_{k \in \mathbf{Z}} \mathbf{a}_{-1, k} \mathbf{f}(2 x, 2 y-k)+\sum_{\mathbf{n} \in \Gamma_{1}} \mathbf{a}_{\mathbf{n}} S(2 \mathbf{x}-\mathbf{n})+\sum_{\mathbf{m} \in \Gamma_{2}} \mathbf{a}_{\mathbf{m}} T(2 \mathbf{x}-\widetilde{\mathbf{m}}), \\
\mathbf{f}(\mathbf{x})=\sum_{k \in \mathbf{Z}} \mathbf{b}_{0, k} \boldsymbol{\varphi}(2 x, 2 y-k)+\sum_{k \in \mathbf{Z}} \mathbf{b}_{-1, k} \mathbf{f}(2 x, 2 y-k)+\sum_{\mathbf{n} \in \Gamma_{1}} \mathbf{b}_{\mathbf{n}} S(2 \mathbf{x}-\mathbf{n}), \\
\mathbf{g}(\mathbf{x})=\sum_{k \in \mathbf{Z}} \mathbf{d}_{0, k} \boldsymbol{\varphi}(2 x, 2 y-k)+\sum_{k \in \mathbf{Z}} \mathbf{d}_{1, k} \mathbf{g}(2 x, 2 y-k)+\sum_{\mathbf{m} \in \Gamma_{2}} \mathbf{d}_{\mathbf{m}} T(2 \mathbf{x}-\widetilde{\mathbf{m}}),
\end{gathered}
$$

where $\mathbf{x}=(x, y)$, and $\mathbf{a}_{j, k}, \mathbf{b}_{j, k}, \mathbf{d}_{j, k}$ are some $r \times r$ matrixes with finitely many nonzeroes.

Denote $\Phi:=\left[\varphi_{0}, \varphi_{1}, \cdots, \varphi_{r-1}, f_{0}, f_{1}, \cdots, f_{r-1}, g_{0}, g_{1}, \cdots, g_{r-1}\right]^{T}$. Then equations (2.10)-(2.12) can be written as a nonhomogeneous refinement 
equation:

$$
\Phi(x, y)=\sum_{k \in \mathbf{Z}} H_{k} \Phi(2 x, 2 y-k)+N(x, y)
$$

where

$$
H_{k}=\left[\begin{array}{ccc}
\mathbf{a}_{0, k} & \mathbf{a}_{-1, k} & \mathbf{a}_{1, k} \\
\mathbf{b}_{0, k} & \mathbf{b}_{-1, k} & 0 \\
\mathbf{d}_{0, k} & 0 & \mathbf{d}_{1, k}
\end{array}\right], \quad N(\mathbf{x})=\left[\begin{array}{c}
N_{1}(2 \mathbf{x}) \\
N_{2}(2 \mathbf{x}) \\
N_{3}(2 \mathbf{x})
\end{array}\right]
$$

with

$$
\begin{aligned}
& N_{1}(x, y):=\sum_{\mathbf{n} \in \Gamma_{1}} \mathbf{a}_{\mathbf{n}} S(2 \mathbf{x}-\mathbf{n})+\sum_{\mathbf{m} \in \Gamma_{2}} \mathbf{a}_{\mathbf{m}} T(2 \mathbf{x}-\widetilde{\mathbf{m}}), \\
& N_{2}(x, y):=\sum_{\mathbf{n} \in \Gamma_{1}} \mathbf{b}_{\mathbf{n}} S(2 \mathbf{x}-\mathbf{n}), N_{3}(x, y):=\sum_{\mathbf{m} \in \Gamma_{2}} \mathbf{d}_{\mathbf{m}} T(2 \mathbf{x}-\widetilde{\mathbf{m}}) .
\end{aligned}
$$

Let $F(\mathbf{x})$ be a function in $V_{0}$. Suppose

$$
F(\mathbf{x})=\sum_{k \in \mathbf{Z}}\left[\mathbf{v}_{0, k}^{0}, \mathbf{v}_{-1, k}^{0}, \mathbf{v}_{1, k}^{0}\right] \Phi(\mathbf{x}-(0, k))+\sum_{\mathbf{n} \in \Gamma_{1}} \mathbf{v}_{\mathbf{n}}^{0} S(\mathbf{x}-\mathbf{n})+\sum_{\mathbf{m} \in \Gamma_{2}} \mathbf{v}_{\mathbf{m}}^{0} T(\mathbf{x}-\widetilde{\mathbf{m}}) .
$$

Since $V_{0} \subset V_{\ell}, \ell=1,2, \cdots, F(\mathbf{x})$ can be written as

$$
F(\mathbf{x})=\sum_{k \in \mathbf{Z}}\left[\mathbf{v}_{0, k}^{\ell}, \mathbf{v}_{-1, k}^{\ell}, \mathbf{v}_{1, k}^{\ell}\right] \Phi\left(2^{\ell} \mathbf{x}-(0, k)\right)+\sum_{\mathbf{n} \in \Gamma_{1}} \mathbf{v}_{\mathbf{n}}^{\ell} S\left(2^{\ell} \mathbf{x}-\mathbf{n}\right)+\sum_{\mathbf{m} \in \Gamma_{2}} \mathbf{v}_{\mathbf{m}}^{\ell} T\left(2^{\ell} \mathbf{x}-\widetilde{\mathbf{m}}\right) .
$$

The above two equations for $F(\mathbf{x})$ and refinement equations (2.13), (2.1) and (2.2) yield the following quad/triangle subdivision algorithm.

Quad/triangle subdivision algorithm For initial control vectors $\mathbf{v}_{\mathbf{k}}^{0}=\left[v_{\mathbf{k}}^{0}, s_{\mathbf{k}, 1}^{0}, \cdots, s_{\mathbf{k}, r-1}^{0}\right],\left\{H_{k}\right\},\left\{Q_{\mathbf{k}}\right\},\left\{P_{\mathbf{k}}\right\}$ and $\mathbf{a}_{\mathbf{k}}, \mathbf{b}_{\mathbf{k}}, \mathbf{d}_{\mathbf{k}}$ yield a quad/triangle subdivision with the vectors $\mathbf{v}_{\mathbf{k}}^{\ell+1}=\left[v_{\mathbf{k}}^{\ell+1}, s_{\mathbf{k}, 1}^{\ell+1}, \cdots, s_{\mathbf{k}, r-1}^{\ell+1}\right]$ after $\ell+1$ steps of subdivision iterations given by

$$
\begin{gathered}
{\left[\mathbf{v}_{0, j}^{\ell+1}, \mathbf{v}_{-1, j}^{\ell+1}, \mathbf{v}_{1, j}^{\ell+1}\right]=\sum_{k \in \mathbf{Z}}\left[\mathbf{v}_{0, k}^{\ell}, \mathbf{v}_{-1, k}^{\ell}, \mathbf{v}_{1, k}^{\ell}\right] H_{j-2 k}, j \in \mathbb{Z},} \\
\mathbf{v}_{\mathbf{n}}^{\ell+1}=\sum_{k \in \mathbf{Z}}\left\{\mathbf{v}_{0, k}^{\ell} \mathbf{a}_{\mathbf{n}-(0,2 k)}+\mathbf{v}_{-1, k}^{\ell} \mathbf{b}_{\mathbf{n}-(0,2 k)}\right\} \\
+\sum_{\mathbf{n}^{\prime} \in \Gamma_{1}} \mathbf{v}_{\mathbf{n}^{\prime}}^{\ell} Q_{\mathbf{n}-2 \mathbf{n}^{\prime}}, \mathbf{n} \in \Gamma_{1}, \\
\mathbf{v}_{\mathbf{m}}^{\ell+1}=\sum_{k \in \mathbf{Z}}\left\{\begin{array}{c}
\left.\mathbf{v}_{0, k}^{\ell} \mathbf{a}_{\mathbf{m}-(0,2 k)}+\mathbf{v}_{1, k}^{\ell} \mathbf{d}_{\mathbf{m}-(0,2 k)}\right\} \\
+\sum_{\mathbf{m}^{\prime} \in \Gamma_{2}} \mathbf{v}_{\mathbf{m}^{\prime}}^{\ell} P_{\mathbf{m}-2 \widetilde{\mathbf{m}}^{\prime}}, \mathbf{m} \in \Gamma_{2} .
\end{array}\right.
\end{gathered}
$$


Obviously, $\mathbf{v}_{0, j}^{\ell}$ above are the vectors associated with quad/triangle nodes $\left(0,2^{-\ell} j\right)$ on the $y$-axis, $\mathbf{v}_{-1, j}^{\ell}$ and $\mathbf{v}_{1, j}^{\ell}$ are the vectors associated with nodes $\left(-2^{-\ell}, j 2^{-\ell}\right)$ and $\left(2^{-\ell}, 2^{-\ell}\left(j-\frac{1}{2}\right)\right)$ resp., and $\mathbf{v}_{\mathbf{n}}^{\ell}, \mathbf{n} \in \Gamma_{1}$ and $\mathbf{v}_{\mathbf{m}}^{\ell}, \mathbf{m} \in \Gamma_{2}$ are the vectors associated with quad nodes $2^{-\ell} \mathbf{n}$ and triangle nodes $2^{-\ell} \widetilde{\mathbf{m}}$ resp.

Observe that since there are only finitely many $\mathbf{a}_{\mathbf{k}}, \mathbf{b}_{\mathbf{k}}, \mathbf{d}_{\mathbf{k}}$ are nonzero, for $\mathbf{n}=\left(n_{1}, n_{2}\right) \in \Gamma_{1}$ and $\mathbf{m}=\left(m_{1}, m_{2}\right) \in \Gamma_{2}$ with $-n_{1}, m_{1}$ large enough, the first terms in both of (2.16) and (2.17) are zero. Therefore, the averaging rule (2.16) for the vertices on the left but far from the $y$-axis coincides with the conventional subdivision algorithm with mask $\left\{Q_{\mathbf{k}}\right\}$ given in (1.2), while the averaging rule (2.17) for the vertices on the right but far from the $y$-axis is the ordinary subdivision algorithm with mask $\left\{P_{\mathbf{k}}\right\}$.
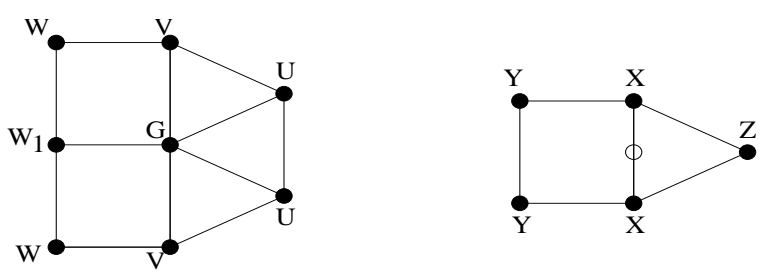

Figure 5: Templates of matrix-valued scheme for "even" vertices (left) and "odd" vertices (right) on the $y$-axis

Quad/triangle subdivision schemes constructed in $[16,10]$ can be derived from nonhomogeneous equations and they can be given in the form of (2.15)(2.17), see [8] for the details. In the following as an example, we give the masks $H_{k}, \mathbf{a}_{\mathbf{k}}, \mathbf{b}_{\mathbf{k}}$ and $\mathbf{d}_{\mathbf{k}}$ for the quad/triangle schemes with the templates in Fig.3 for vertices on the left of the $y$-axis, and the templates in Fig.4 for vertices on the right of the $y$-axis, and the templates in Fig. 5 for vertices on the $y$-axis, where $G, U, V, W, W_{1}, X, Y, Z$ are some $r \times r$ constant matrices. In this case, the corresponding nonzero $H_{k}$ are

$$
\begin{aligned}
& H_{-2}=\left[\begin{array}{ccc}
V, & M, & 0 \\
W, & M, & 0 \\
U, & 0, & C
\end{array}\right], H_{-1}=\left[\begin{array}{ccc}
X, & K, & C \\
Y, & K, & 0 \\
Z, & 0, & B
\end{array}\right] \\
& H_{0}=\left[\begin{array}{ccc}
G, & J, & B \\
W_{1}, & J, & 0 \\
U, & 0, & B
\end{array}\right], H_{1}=\left[\begin{array}{ccc}
X, & K, & B \\
Y, & K, & 0 \\
0, & 0, & C
\end{array}\right], H_{2}=\left[\begin{array}{ccc}
V, & M, & C \\
W, & M, & 0 \\
0, & 0, & 0
\end{array}\right]
\end{aligned}
$$


and the corresponding nonzero $\mathbf{a}_{j, k}, \mathbf{b}_{j, k}, \mathbf{d}_{j, k}$ are

$$
\begin{aligned}
& \mathbf{a}_{2,0}=C, \mathbf{a}_{2,1}=\mathbf{a}_{2,-1}=D, \mathbf{a}_{-2,0}=L, \\
& \mathbf{a}_{-2,1}=\mathbf{a}_{-2,-1}=N, \mathbf{a}_{-2,2}=\mathbf{a}_{-2,-2}=M, \\
& \mathbf{b}_{-2,0}=R, \mathbf{b}_{-2,1}=\mathbf{b}_{-2,-1}=J, \mathbf{b}_{-2,2}=\mathbf{b}_{-2,-2}=L, \\
& \mathbf{b}_{-3,0}=J, \mathbf{b}_{-3,1}=\mathbf{b}_{-3,-1}=K, \mathbf{b}_{-3,2}=\mathbf{b}_{-3,-2}=M, \\
& \mathbf{b}_{-4,0}=L, \mathbf{b}_{-4,1}=\mathbf{b}_{-4,-1}=M, \mathbf{b}_{-4,2}=\mathbf{b}_{-4,-2}=N, \\
& \mathbf{d}_{2,-1}=P, \mathbf{d}_{2,0}=\mathbf{d}_{2,-2}=B, \mathbf{d}_{2,1}=\mathbf{d}_{2,-3}=D, \\
& \mathbf{d}_{3,-1}=\mathbf{d}_{3,-2}=B, \mathbf{d}_{3,0}=\mathbf{d}_{3,-3}=C, \mathbf{d}_{4,-1}=C, \mathbf{d}_{4,0}=\mathbf{d}_{4,-2}=D .
\end{aligned}
$$

This quad/triangle scheme has the same sizes of templates as Stam-Loop's scheme.

\subsection{Limiting surfaces}

In this subsection we consider the limiting surfaces. First, let us discuss the case $r=1$. Let $h(x)=1-|x|$ for $|x| \leq 1$ and $h(x)=0$ for $|x|>1$ be the 1-D hat function. Let $h_{1}(\mathbf{x})=h(x) h(y)$ be the 2-D hat function. See the support of $h_{1}$ in the middle of Fig.6. Let $h_{0}(\mathbf{x})$ be the "hat" function supported on the region shown on the left of Fig. 6 with $h_{0}(0,0)=1, h_{0}(\mathbf{x})$ being a piecewise linear polynomial on each of three triangles on the right of the $y$-axis, and $h_{0}(\mathbf{x})=(1+x) h(y)$ for $\mathbf{x}=(x, y),-1 \leq x \leq 0$. Let $h_{2}(\mathbf{x})$ be the hat function supported on a neighborhood of $(0,0)$ consisting of 6 triangles shown on the right of Fig. 6 with $h_{2}(0,0)=1, h_{2}(\mathbf{x})$ being a piecewise linear polynomial on each of these six triangles.
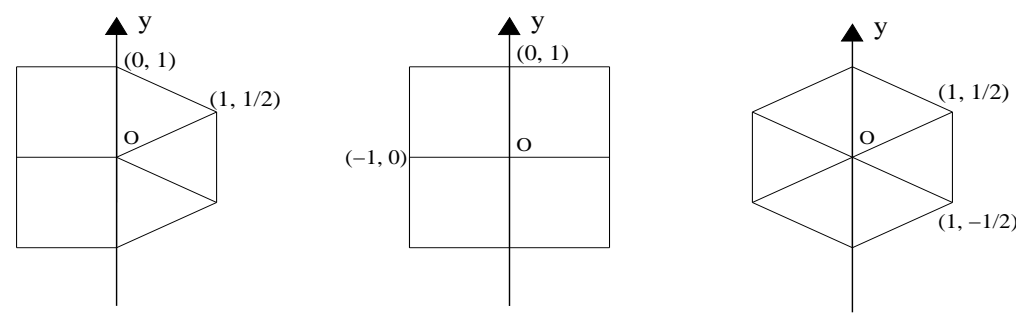

Figure 6: Supports of hat functions $h_{0}(\mathbf{x}), h_{1}(\mathbf{x}), h_{2}(\mathbf{x})$

For a conventional dyadic (scalar-valued) quad or triangle subdivision scheme with refinement mask $\left\{p_{\mathbf{k}}\right\}$, let $v_{\mathbf{k}}^{\ell}$ be the vertices of the refined mesh after the subdivision is applied $\ell$ times to an initial regular mesh with vertices $v_{\mathbf{k}}^{0}$. Then, for sufficiently large values of $\ell, v_{\mathbf{k}}^{\ell}$ provide an accurate discrete approximation of the limiting surface. More precisely, let $\sum_{\mathbf{k}} v_{\mathbf{k}}^{\ell} \mathcal{C}_{0}\left(2^{\ell} \mathbf{x}-\mathbf{k}\right)$ 
be the polyhedron (refined mesh) with vertices $v_{\mathbf{k}}^{\ell}$, where $\mathcal{C}_{0}(\mathbf{x})$ is a suitable hat function. (One can choose $\mathcal{C}_{0}(\mathbf{x})$ to be $h_{1}(\mathbf{x})$ for the quad subdivision and $\mathcal{C}_{0}(\mathbf{x})$ to be $h_{2}(\mathbf{x})$ for the triangle subdivision.) Then the sequence of finer and finer polyhedra gives an approximation to the limiting surface given by $\sum_{\mathbf{k}} v_{\mathbf{k}}^{0} \phi(\mathbf{x}-\mathbf{k})$, where $\phi$ is the refinable function associated with $\left\{p_{\mathbf{k}}\right\}$, see e.g. [4]. Next, we consider the limiting surface of a quad/triangle subdivision.

Let $v_{\mathbf{k}}^{\ell}$ be the vertices of the refined mesh given by $(2.15)-(2.17)$ after $\ell$ steps of quad/triangle subdivision iterations with masks $\left\{H_{k}\right\}_{k \in \mathbf{Z}},\left\{q_{\mathbf{k}}\right\}_{\mathbf{k} \in \mathbf{Z}^{2}}$, $\left\{p_{\mathbf{k}}\right\}_{\mathbf{k} \in \mathbf{Z}^{2}}$ and $a_{\mathbf{k}}, b_{\mathbf{k}}, d_{\mathbf{k}}$ applied to an initial control net with vertices $v_{\mathbf{k}}^{0}$. We say this scheme is $L^{\infty}$-convergent if for any $v_{\mathbf{k}}^{0}$, there is a continuous function $F(\mathbf{x})$ on $\mathbb{R}^{2}$ such that $F \not \equiv 0$ for at least one $\left\{v_{\mathbf{k}}^{0}\right\}_{\mathbf{k}}$, and that for any $R>0$,

$$
\left\{\left\|v_{\mathbf{k}}^{\ell}-F\left(\frac{\mathbf{k}}{2^{\ell}}\right)\right\|_{\infty,\|\mathbf{k}\| \leq 2^{\ell} R, k_{1} \leq 0}+\left\|v_{\mathbf{k}}^{\ell}-F\left(\frac{\widetilde{\mathbf{k}}}{2^{\ell}}\right)\right\|_{\infty,\|\mathbf{k}\| \leq 2^{\ell} R, k_{1}>0}\right\} \rightarrow 0,
$$

as $\ell \rightarrow \infty$. Next we show that the limiting function (surface) $F(\mathbf{x})$ is given by a linear combination of $S(\mathbf{x}), T(\mathbf{x}), \Phi(\mathbf{x})$ and their integer-shifts, where $S(\mathbf{x}), T(\mathbf{x})$ are the compactly supported refinable functions associated with $\left\{q_{\mathbf{k}}\right\}$ and $\left\{p_{\mathbf{k}}\right\}$ resp., and $\Phi=[\varphi, f, g]^{T}$ be the refinable function vector satisfying the nonhomogeneous refinement equation (2.13). To this regard, we consider the cascade operator $S_{H, N}$ associated with (2.13) defined by

$$
S_{H, N} G(\mathbf{x}):=\sum_{k \in \mathbf{Z}} H_{k} G(\mathbf{x}-(0, k))+N(\mathbf{x})
$$

for $G(\mathbf{x})=\left[g_{1}(\mathbf{x}), g_{2}(\mathbf{x}), g_{3}(\mathbf{x})\right]^{T}$, where $N(\mathbf{x})$ is the nonhomogeneous term in (2.13). Clearly if $\left\{\left(S_{H, N}\right)^{n} G(\mathbf{x})\right\}_{n}$, which is called the cascade algorithm sequence, converges pointwise or in $L^{2}$-norm, then its limit is $\Phi$, the refinable function satisfying (2.13). Let

$$
\Phi_{0}(\mathbf{x}):=\left[h_{0}(\mathbf{x}), h_{1}(\mathbf{x}+(1,0)), h_{2}\left(\mathbf{x}-\left(1,-\frac{1}{2}\right)\right)\right]^{T},
$$

where $h_{0}, h_{1}, h_{2}$ are the hat functions mentioned above. Then for $\ell=0,1, \cdots$, the polyhedron with vertices $v_{\mathbf{k}}^{\ell}$, the refined quad/triangle mesh after $\ell$ steps of iterations, is given by

$$
\begin{aligned}
L_{\ell}(\mathbf{x}):=\sum_{k \in \mathbf{Z}}\left[v_{0, k}^{\ell}, v_{-1, k}^{\ell}, v_{1, k}^{\ell}\right] \Phi_{0}\left(2^{\ell} x, 2^{\ell} y-k\right) & \\
& +\sum_{\mathbf{n} \in \Gamma_{1}} v_{\mathbf{n}}^{\ell} h_{1}\left(2^{\ell} \mathbf{x}-\mathbf{n}\right)+\sum_{\mathbf{m} \in \Gamma_{2}} v_{\mathbf{m}}^{\ell} h_{2}\left(2^{\ell} \mathbf{x}-\widetilde{\mathbf{m}}\right) .
\end{aligned}
$$


Proposition 1. Suppose a quad/triangle scheme with mask $\left\{H_{k}\right\}_{k \in \mathbf{Z}},\left\{q_{\mathbf{k}}\right\}_{\mathbf{k} \in \mathbf{Z}^{2}}$, $\left\{p_{\mathbf{k}}\right\}_{\mathbf{k} \in \mathbf{Z}^{2}}$ and $a_{\mathbf{k}}, b_{\mathbf{k}}, d_{\mathbf{k}}$ are $L^{\infty}$-convergent. If in addition, the cascade algorithm sequence $\left\{\left(S_{H, N}\right)^{n} \Phi_{0}(\mathbf{x})\right\}_{n}$ converges pointwise, then the sequence $\left\{L_{\ell}(\mathbf{x})\right\}_{\ell}$ defined by (2.22) converges pointwise to

$$
L(\mathbf{x})=\sum_{k \in \mathbf{Z}}\left[v_{0, k}^{0}, v_{-1, k}^{0}, v_{1, k}^{0}\right] \Phi(x, y-k)+\sum_{\mathbf{n} \in \Gamma_{1}} v_{\mathbf{n}}^{0} S(\mathbf{x}-\mathbf{n})+\sum_{\mathbf{m} \in \Gamma_{2}} v_{\mathbf{m}}^{0} T(\mathbf{x}-\tilde{\mathbf{m}}) .
$$

Proof. Since this quad/triangle scheme is $L^{\infty}$-convergent, the schemes with masks $\left\{q_{\mathbf{k}}\right\}_{\mathbf{k} \in \mathbf{Z}^{2}},\left\{p_{\mathbf{k}}\right\}_{\mathbf{k} \in \mathbf{Z}^{2}}$ used for quad vertices and triangle vertices are also $L^{\infty}$-convergent. Thus both $\sum_{\mathbf{n} \in \Gamma_{1}} v_{\mathbf{n}}^{\ell} S\left(2^{\ell} \mathbf{x}-\mathbf{n}\right)-\sum_{\mathbf{n} \in \Gamma_{1}} v_{\mathbf{n}}^{\ell} h_{1}\left(2^{\ell} \mathbf{x}-\mathbf{n}\right)$ and $\sum_{\mathbf{m} \in \Gamma_{2}} v_{\mathbf{m}}^{\ell} T\left(2^{\ell} \mathbf{x}-\widetilde{\mathbf{m}}\right)-\sum_{\mathbf{m} \in \Gamma_{2}} v_{\mathbf{m}}^{\ell} h_{2}\left(2^{\ell} \mathbf{x}-\widetilde{\mathbf{m}}\right)$ approach to zero as $\ell \rightarrow \infty$. Therefore, it is enough to show that

$$
\begin{aligned}
\widetilde{L}_{\ell}(\mathbf{x}):=\sum_{k \in \mathbf{Z}}\left[v_{0, k}^{\ell}, v_{-1, k}^{\ell}, v_{1, k}^{\ell}\right] \Phi_{0}\left(2^{\ell} x, 2^{\ell} y-k\right) & \\
& +\sum_{\mathbf{n} \in \Gamma_{1}} v_{\mathbf{n}}^{\ell} S\left(2^{\ell} \mathbf{x}-\mathbf{n}\right)+\sum_{\mathbf{m} \in \Gamma_{2}} v_{\mathbf{m}}^{\ell} T\left(2^{\ell} \mathbf{x}-\widetilde{\mathbf{m}}\right),
\end{aligned}
$$

converges pointwise to $L(\mathbf{x})$ in $(2.23)$.

Denote $\mathbf{w}_{k}^{\ell}:=\left[\begin{array}{lll}v_{0, k}^{\ell}, & v_{-1, k}^{\ell}, & v_{1, k}^{\ell}\end{array}\right], \quad \ell=0,1, \cdots$. From the subdivision algorithm (2.15)-(2.17), we have

$$
\begin{aligned}
& \sum_{k \in \mathbf{Z}} \mathbf{w}_{k}^{1} \Phi(2 x, 2 y-k)+\sum_{\mathbf{n} \in \Gamma_{1}} v_{\mathbf{n}}^{1} S(2 \mathbf{x}-\mathbf{n})+\sum_{\mathbf{m} \in \Gamma_{2}} v_{\mathbf{m}}^{1} T(2 \mathbf{x}-\widetilde{\mathbf{m}}) \\
& =\sum_{k \in \mathbf{Z}} \mathbf{w}_{k}^{0} \Phi(x, y-k)+\sum_{\mathbf{n} \in \Gamma_{1}} v_{\mathbf{n}}^{0} S(\mathbf{x}-\mathbf{n})+\sum_{\mathbf{m} \in \Gamma_{2}} v_{\mathbf{m}}^{0} T(\mathbf{x}-\widetilde{\mathbf{m}}) .
\end{aligned}
$$

On the other hand, for any $G(\mathbf{x})=\left[g_{1}(\mathbf{x}), g_{2}(\mathbf{x}), g_{3}(\mathbf{x})\right]^{T}$,

$$
\begin{aligned}
& \sum_{k \in \mathbf{Z}} \mathbf{w}_{k}^{0} S_{H, N} G(x, y-k)=\sum_{k \in \mathbf{Z}} \mathbf{w}_{k}^{0}\left\{\sum_{j \in \mathbf{Z}} H_{j} G(2 x, 2 y-2 k-j)+N(x, y-k)\right\} \\
& =\sum_{j \in \mathbf{Z}} \sum_{k \in \mathbf{Z}} \mathbf{w}_{k}^{0} H_{j-2 k} G(2 x, 2 y-j)+\sum_{k \in \mathbf{Z}} \mathbf{w}_{k}^{0} N(x, y-k) \\
& =\sum_{j \in \mathbf{Z}} \mathbf{w}_{j}^{1} G(2 x, 2 y-j)+\sum_{k \in \mathbf{Z}} \mathbf{w}_{k}^{0} N(x, y-k) .
\end{aligned}
$$

In particular, when $G=\Phi$, since $S_{H, N} \Phi=\Phi$, we have

$$
\sum_{k \in \mathbf{Z}} \mathbf{w}_{k}^{0} \Phi(x, y-k)=\sum_{j \in \mathbf{Z}} \mathbf{w}_{j}^{1} \Phi(2 x, 2 y-j)+\sum_{k \in \mathbf{Z}} \mathbf{w}_{k}^{0} N(x, y-k) .
$$


This and (2.24) imply that

$$
\begin{aligned}
& \sum_{\mathbf{n} \in \Gamma_{1}} v_{\mathbf{n}}^{1} S(2 \mathbf{x}-\mathbf{n})+\sum_{\mathbf{m} \in \Gamma_{2}} v_{\mathbf{m}}^{1} T(2 \mathbf{x}-\widetilde{\mathbf{m}}) \\
& =\sum_{k \in \mathbf{Z}} \mathbf{w}_{k}^{0} N(x, y-k)+\sum_{\mathbf{n} \in \Gamma_{1}} v_{\mathbf{n}}^{0} S(\mathbf{x}-\mathbf{n})+\sum_{\mathbf{m} \in \Gamma_{2}} v_{\mathbf{m}}^{0} T(\mathbf{x}-\widetilde{\mathbf{m}}),
\end{aligned}
$$

which leads that

$$
\begin{aligned}
& \widetilde{L}_{1}(\mathbf{x})=\sum_{k \in \mathbf{Z}} \mathbf{w}_{k}^{1} \Phi_{0}(2 x, 2 y-k)+\sum_{\mathbf{n} \in \Gamma_{1}} v_{\mathbf{n}}^{1} S(2 \mathbf{x}-\mathbf{n})+\sum_{\mathbf{m} \in \Gamma_{2}} v_{\mathbf{m}}^{1} T(2 \mathbf{x}-\widetilde{\mathbf{m}}) \\
& =\sum_{k \in \mathbf{Z}} \mathbf{w}_{k}^{1} \Phi_{0}(2 x, 2 y-k)+\sum_{k \in \mathbf{Z}} \mathbf{w}_{k}^{0} N(x, y-k)+\sum_{\mathbf{n} \in \Gamma_{1}} v_{\mathbf{n}}^{0} S(\mathbf{x}-\mathbf{n})+\sum_{\mathbf{m} \in \Gamma_{2}} v_{\mathbf{m}}^{0} T(\mathbf{x}-\widetilde{\mathbf{m}}) \\
& =\sum_{k \in \mathbf{Z}} \mathbf{w}_{k}^{0}\left(S_{H, N} \Phi_{0}\right)(x, y-k)+\sum_{\mathbf{n} \in \Gamma_{1}} v_{\mathbf{n}}^{0} S(\mathbf{x}-\mathbf{n})+\sum_{\mathbf{m} \in \Gamma_{2}} v_{\mathbf{m}}^{0} T(\mathbf{x}-\widetilde{\mathbf{m}}) .
\end{aligned}
$$

Similarly, we have for $\ell>1$,

$$
\begin{aligned}
& \widetilde{L}_{\ell}(\mathbf{x})=\sum_{k \in \mathbf{Z}} \mathbf{w}_{k}^{\ell} \Phi_{0}\left(2^{\ell} x, 2^{\ell} y-k\right)+\sum_{\mathbf{n} \in \Gamma_{1}} v_{\mathbf{n}}^{\ell} S\left(2^{\ell} \mathbf{x}-\mathbf{n}\right)+\sum_{\mathbf{m} \in \Gamma_{2}} v_{\mathbf{m}}^{\ell} T\left(2^{\ell} \mathbf{x}-\widetilde{\mathbf{m}}\right) \\
& =\sum_{k \in \mathbf{Z}} \mathbf{w}_{k}^{0}\left(S_{H, N}\right)^{\ell} \Phi_{0}(x, y-k)+\sum_{\mathbf{n} \in \Gamma_{1}} v_{\mathbf{n}}^{0} S(\mathbf{x}-\mathbf{n})+\sum_{\mathbf{m} \in \Gamma_{2}} v_{\mathbf{m}}^{0} T(\mathbf{x}-\widetilde{\mathbf{m}}) .
\end{aligned}
$$

Therefore, if $\left\{\left(S_{H, N}\right)^{\ell} \Phi_{0}(\mathbf{x})\right\}_{\ell}$ converges (to $\left.\Phi\right)$, then $\widetilde{L}_{\ell}(\mathbf{x})$ is convergent with limit $\sum_{k \in \mathbf{Z}} \mathbf{w}_{k}^{0} \Phi(x, y-k)+\sum_{\mathbf{n} \in \Gamma_{1}} v_{\mathbf{n}}^{0} S(\mathbf{x}-\mathbf{n})+\sum_{\mathbf{m} \in \Gamma_{2}} v_{\mathbf{m}}^{0} T(\mathbf{x}-\widetilde{\mathbf{m}})$, as desired.

Next, we consider the limiting surface of a matrix-valued quad/triangle subdivision scheme. First we recall some results on the limiting surfaces generated by a conventional matrix-valued subdivision. Assume that $\left\{G_{\mathbf{k}}\right\}_{\mathbf{k}}$ is a matrix-valued refinement mask (for either quad or triangle subdivision) and $\phi=\left[\phi_{0}, \cdots, \phi_{r-1}\right]^{T}$ is the associated refinable function vector satisfying (2.3) with $\mathbf{y}_{0}$ given in (2.4). Let $\mathbf{v}_{\mathbf{k}}^{\ell}=\left[v_{\mathbf{k}}^{\ell}, s_{\mathbf{k}, 1}^{\ell}, \cdots, s_{\mathbf{k}, r-1}^{\ell}\right]$ be the vectors given by (2.5) from initial control vectors $\mathbf{v}_{\mathbf{k}}^{0}$. Then for sufficiently large values of $\ell$, the refined mesh with $v_{\mathbf{k}}^{\ell}$, the first component of $\mathbf{v}_{\mathbf{k}}^{\ell}$, provides an accurate discrete approximation of the target subdivision surface. More precisely, let $\sum_{\mathbf{k}} v_{\mathbf{k}}^{\ell} \mathcal{C}_{0}\left(2^{\ell} \mathbf{x}-\mathbf{k}\right)$ be the polyhedron with vertices $v_{\mathbf{k}}^{\ell}$, where $\mathcal{C}_{0}(\mathbf{x})$ is a suitable hat function (again, one can choose $\mathcal{C}_{0}(\mathbf{x})$ to be $h_{1}(\mathbf{x})$ 
for quad subdivision and $\mathcal{C}_{0}(\mathbf{x})$ to be $h_{2}(\mathbf{x})$ for triangle subdivision). Then under certain mild condition, the sequence of finer and finer polyhedra gives an approximation to the limiting surface given by

$$
\sum_{\mathbf{k}}\left\{v_{\mathbf{k}}^{0} \phi_{0}(\mathbf{x}-\mathbf{k})+s_{\mathbf{k}, 1}^{0} \phi_{1}(\mathbf{x}-\mathbf{k})+\cdots+s_{\mathbf{k}, r-1}^{0} \phi_{r-1}(\mathbf{x}-\mathbf{k})\right\} .
$$

The reader refers to [4] for the detailed discussion on the $L_{2}$ convergence. Following the discussion in [4], one can show that for each $j, 1 \leq j \leq r-1$, $\sum_{\mathbf{k}} s_{\mathbf{k}, j}^{\ell} \mathcal{C}_{0}\left(2^{\ell} \mathbf{x}-\mathbf{k}\right.$ ), the polyhedron with vertices $s_{\mathbf{k}, j}^{\ell}$ (another component of $\left.\mathbf{v}_{\mathbf{k}}^{\ell}\right)$, and even $\sum_{\mathbf{k}} s_{\mathbf{k}, j}^{\ell} \mathcal{D}_{0}\left(2^{\ell} \mathbf{x}-\mathbf{k}\right)$, where $\mathcal{D}_{0}$ is a compactly supported function, converge to the zero function.

Now let us consider the limiting surface of a matrix-valued quad/triangle subdivision. For control vectors $\mathbf{v}_{\mathbf{k}}^{0}=\left[v_{\mathbf{k}}^{0}, s_{\mathbf{k}, 1}^{0}, \cdots, s_{\mathbf{k}, r-1}^{0}\right]$, let $\mathbf{v}_{\mathbf{k}}^{\ell}=\left[v_{\mathbf{k}}^{\ell}, s_{\mathbf{k}, 1}^{\ell}\right.$, $\left.\cdots, s_{\mathbf{k}, r-1}^{\ell}\right]$ be the subdivided vectors given by (2.15)-(2.17) after $\ell$ steps of a quad/triangle subdivision iterations with mask $H=\left\{H_{k}\right\}_{k \in \mathbf{Z}},\left\{Q_{\mathbf{k}}\right\}_{\mathbf{k} \in \mathbf{Z}^{2}}$, $\left\{P_{\mathbf{k}}\right\}_{\mathbf{k} \in \mathbf{Z}^{2}}$ and $\mathbf{a}_{\mathbf{k}}, \mathbf{b}_{\mathbf{k}}, \mathbf{d}_{\mathbf{k}}$. We say this quad/triangle scheme is $L^{\infty}$-convergent if for any $\mathbf{v}_{\mathbf{k}}^{0}$, there is a continuous function vector $F=\left[F_{0}, F_{1}, \cdots, F_{r-1}\right]^{T}$ on $\mathbb{R}^{2}$ such that (2.20) with $v_{\mathbf{k}}^{0}$ replaced by $\mathbf{v}_{\mathbf{k}}^{0}$ holds. From the above discussion, the condition that the associated $S(\mathbf{x}), T(\mathbf{x})$ satisfy (2.3) with $\mathbf{y}_{0}$ given by (2.4) implies $F_{j}=0$ for $1 \leq j \leq r-1$. Thus as in a conventional matrixvalued subdivision, when we use matrix-valued masks for quad/triangle subdivision, we should use the first components $v_{\mathbf{k}}^{0}$ of $\mathbf{v}_{\mathbf{k}}^{0}$ as the vertices of the refined meshes. Clearly, such refined meshes can be expressed as

$$
\begin{aligned}
& L_{\ell}(\mathbf{x}):=\sum_{k \in \mathbf{Z}}\left\{v_{0, k}^{\ell} h_{0}\left(2^{\ell} \mathbf{x}-(0, k)\right)+v_{-1, k}^{\ell} h_{1}\left(2^{\ell} \mathbf{x}-(-1, k)\right)\right. \\
& \left.+v_{1, k}^{\ell} h_{2}\left(2^{\ell} \mathbf{x}-\left(1, k-\frac{1}{2}\right)\right)\right\}+\sum_{\mathbf{n} \in \Gamma_{1}} v_{\mathbf{n}}^{\ell} h_{1}\left(2^{\ell} \mathbf{x}-\mathbf{n}\right)+\sum_{\mathbf{m} \in \Gamma_{2}} v_{\mathbf{m}}^{\ell} h_{2}\left(2^{\ell} \mathbf{x}-\widetilde{\mathbf{m}}\right) .
\end{aligned}
$$

Next proposition shows that the limiting surface of the sequence of these finer and finer polyhedra is given as the linear combination of the integershifts of $S, T$ and $\Phi$, where $S=\left[S_{0}, \cdots, S_{r-1}\right]^{T}$ and $T=\left[T_{0}, \cdots, T_{r-1}\right]^{T}$ are the compactly supported refinable function vectors associated with $\left\{Q_{\mathbf{k}}\right\}$ and $\left\{P_{\mathbf{k}}\right\}$ resp., and $\Phi=\left[\varphi_{0}, \cdots, \varphi_{r-1}, f_{0}, \cdots, f_{r-1}, g_{0}, \cdots, g_{r-1}\right]^{T}$ is the associated refinable function vector satisfying the nonhomogeneous refinement equation (2.13). Again, to this regard, we introduce the cascade operator $S_{H, N}$ associated with (2.13) defined by

$$
S_{H, N} G(\mathbf{x}):=\sum_{k \in \mathbf{Z}} H_{k} G(\mathbf{x}-(0, k))+N(\mathbf{x}),
$$


for $G(\mathbf{x})=\left[g_{1}(\mathbf{x}), \cdots, g_{3 r}(\mathbf{x})\right]^{T}$. Clearly if $\left\{\left(S_{H, N}\right)^{n} G(\mathbf{x})\right\}_{n}$ converges (pointwise or in $L^{2}$ norm), then its limit is $\Phi$, the refinable function vector satisfies (2.13). Denote

$$
\Phi_{0}(\mathbf{x}):=\left[h_{0}(\mathbf{x}), 0, \cdots, 0, h_{1}(\mathbf{x}+(1,0)), 0, \cdots, 0, h_{2}\left(\mathbf{x}-\left(1,-\frac{1}{2}\right)\right), 0, \cdots, 0\right]^{T}
$$

with the 1st, $(r+1)$-th and $(2 r+1)$-th components to be $h_{0}(\mathbf{x}), h_{1}(\mathbf{x}+(1,0))$ and $h_{2}\left(\mathbf{x}-\left(1,-\frac{1}{2}\right)\right)$ resp., where $h_{0}, h_{1}$ and $h_{2}$ are the hat functions defined above.

Proposition 2. Suppose a quad/triangle scheme with matrix-valued mask $\left\{H_{k}\right\}_{k \in \mathbf{Z}},\left\{Q_{\mathbf{k}}\right\}_{\mathbf{k} \in \mathbf{Z}^{2}},\left\{P_{\mathbf{k}}\right\}_{\mathbf{k} \in \mathbf{Z}^{2}}$ and $\mathbf{a}_{\mathbf{k}}, \mathbf{b}_{\mathbf{k}}, \mathbf{d}_{\mathbf{k}}$ are $L^{\infty}$-convergent. If in addition, the cascade algorithm sequence $\left\{\left(S_{H, N}\right)^{n} \Phi_{0}(\mathbf{x})\right\}_{n}$ converges pointwise, then the sequence $\left\{L_{\ell}(\mathbf{x})\right\}_{\ell}$ defined by (2.25) converges pointwise to

$$
L(\mathbf{x})=\sum_{k \in \mathbf{Z}}\left[\mathbf{v}_{0, k}^{0}, \mathbf{v}_{-1, k}^{0}, \mathbf{v}_{1, k}^{0}\right] \Phi(x, y-k)+\sum_{\mathbf{n} \in \Gamma_{1}} \mathbf{v}_{\mathbf{n}}^{0} S(\mathbf{x}-\mathbf{n})+\sum_{\mathbf{m} \in \Gamma_{2}} \mathbf{v}_{\mathbf{m}}^{0} T(\mathbf{x}-\widetilde{\mathbf{m}}) .
$$

Proof. Since this quad/triangle scheme is $L^{\infty}$-convergent, the schemes with masks $\left\{Q_{\mathbf{k}}\right\}_{\mathbf{k} \in \mathbf{Z}^{2}},\left\{P_{\mathbf{k}}\right\}_{\mathbf{k} \in \mathbf{Z}^{2}}$ used for quad vertices and triangle vertices are also $L^{\infty}$-convergent. Thus, both $\sum_{\mathbf{n} \in \Gamma_{1}} v_{\mathbf{n}}^{\ell} S_{0}\left(2^{\ell} \mathbf{x}-\mathbf{n}\right)-\sum_{\mathbf{n} \in \Gamma_{1}} v_{\mathbf{n}}^{\ell} h_{1}\left(2^{\ell} \mathbf{x}-\right.$ $\mathbf{n})$ and $\sum_{\mathbf{m} \in \Gamma_{2}} v_{\mathbf{m}}^{\ell} T_{0}\left(2^{\ell} \mathbf{x}-\widetilde{\mathbf{m}}\right)-\sum_{\mathbf{m} \in \Gamma_{2}} v_{\mathbf{m}}^{\ell} h_{2}\left(2^{\ell} \mathbf{x}-\widetilde{\mathbf{m}}\right)$ approach to zero as $\ell \rightarrow \infty$. Furthermore, for $1 \leq j \leq r-1, \sum_{\mathbf{n} \in \Gamma_{1}} s_{\mathbf{n}, j}^{\ell} S_{j}\left(2^{\ell} \mathbf{x}-\mathbf{n}\right)$ and $\sum_{\mathbf{m} \in \Gamma_{2}} s_{\mathbf{m}, j}^{\ell} T_{j}\left(2^{\ell} \mathbf{x}-\widetilde{\mathbf{m}}\right)$ approach to zero as $\ell \rightarrow \infty$. Therefore, it is enough to show that

$$
\begin{aligned}
\widetilde{L}_{\ell}(\mathbf{x}):=\sum_{k \in \mathbf{Z}}\left[\mathbf{v}_{0, k}^{\ell}, \mathbf{v}_{-1, k}^{\ell}, \mathbf{v}_{1, k}^{\ell}\right] \Phi_{0}\left(2^{\ell} x, 2^{\ell} y-k\right) \\
+\sum_{\mathbf{n} \in \Gamma_{1}} \mathbf{v}_{\mathbf{n}}^{\ell} S\left(2^{\ell} \mathbf{x}-\mathbf{n}\right)+\sum_{\mathbf{m} \in \Gamma_{2}} \mathbf{v}_{\mathbf{m}}^{\ell} T\left(2^{\ell} \mathbf{x}-\widetilde{\mathbf{m}}\right),
\end{aligned}
$$

converges pointwise to $L(\mathbf{x})$ in (2.27). Indeed, with $\mathbf{w}_{k}^{\ell}:=\left[\mathbf{v}_{0, k}^{\ell}, \mathbf{v}_{-1, k}^{\ell}, \mathbf{v}_{1, k}^{\ell}\right]$, $\ell=0,1, \cdots$, one can obtain as in the proof of Proposition 1 that for $\ell \geq 1$,

$$
\begin{aligned}
\widetilde{L}_{\ell}(\mathbf{x}) & =\sum_{k \in \mathbf{Z}} \mathbf{w}_{k}^{\ell} \Phi_{0}\left(2^{\ell} x, 2^{\ell} y-k\right)+\sum_{\mathbf{n} \in \Gamma_{1}} \mathbf{v}_{\mathbf{n}}^{\ell} S\left(2^{\ell} \mathbf{x}-\mathbf{n}\right)+\sum_{\mathbf{m} \in \Gamma_{2}} \mathbf{v}_{\mathbf{m}}^{\ell} T\left(2^{\ell} \mathbf{x}-\widetilde{\mathbf{m}}\right) \\
& =\sum_{k \in \mathbf{Z}} \mathbf{w}_{k}^{0}\left(S_{H, N}\right)^{\ell} \Phi_{0}(x, y-k)+\sum_{\mathbf{n} \in \Gamma_{1}} \mathbf{v}_{\mathbf{n}}^{0} S(\mathbf{x}-\mathbf{n})+\sum_{\mathbf{m} \in \Gamma_{2}} \mathbf{v}_{\mathbf{m}}^{0} T(\mathbf{x}-\widetilde{\mathbf{m}}) .
\end{aligned}
$$

Therefore, if $\left\{\left(S_{H, N}\right)^{\ell} \Phi_{0}(\mathbf{x})\right\}_{\ell}$ converges pointwise (to $\left.\Phi\right)$, then $\widetilde{L}_{\ell}(\mathbf{x})$ is also convergent pointwise with limit $\sum_{k \in \mathbf{Z}} \mathbf{w}_{k}^{0} \Phi(x, y-k)+\sum_{\mathbf{n} \in \Gamma_{1}} \mathbf{v}_{\mathbf{n}}^{0} S(\mathbf{x}-\mathbf{n})+$ $\sum_{\mathbf{m} \in \Gamma_{2}} \mathbf{v}_{\mathbf{m}}^{0} T(\mathbf{x}-\widetilde{\mathbf{m}})$, as desired. 


\section{Polynomial reproduction}

Polynomial reproduction, or polynomial preservation is an important property for a subdivision scheme. Under certain condition, for example, the stability of the associated refinable function, a scheme's reproduction of polynomials of (total) degree at least $m$ is a necessary condition for its $C^{m}$ smoothness. Polynomial reproduction of quad/triangle schemes is studied in [9]. As shown in [10], the result on the polynomial reproduction in [9] is critical for the smoothness analysis and construction of quad/triangle schemes. In this section, we study polynomial reproduction of matrix-valued quad/triangle schemes. Since both schemes are associated with a nonhomogeneous refinement equation, we can treat this issue for either scalar-valued or matrix-valued quad/triangle schemes in a uniform way.

Let $\left\{H_{k}\right\}_{k},\left\{Q_{\mathbf{k}}\right\}_{\mathbf{k}},\left\{P_{\mathbf{k}}\right\}_{\mathbf{k}}$ and $\mathbf{a}_{\mathbf{k}}, \mathbf{b}_{\mathbf{k}}, \mathbf{d}_{\mathbf{k}}$ be the masks for a quad/triangle scheme, where $H_{k}$ are $3 r \times 3 r$ matrices, $Q_{\mathbf{k}}, P_{\mathbf{k}}$ and $\mathbf{a}_{\mathbf{k}}, \mathbf{b}_{\mathbf{k}}, \mathbf{d}_{\mathbf{k}}$ are $r \times r$ matrices. Assume that the masks $\left\{Q_{\mathbf{k}}\right\}_{\mathbf{k}}$ and $\left\{P_{\mathbf{k}}\right\}_{\mathbf{k}}$ for quad vertices and triangle vertices have sum rule of order (at least) $m+1$. The reader sees the definition of sum rule order for a 1-D matrix-valued mask below, and refers to e.g. $[1,7,6]$ for the definition of sum rule order for a 2-D matrix-valued mask. Throughout this section, a Greek letter such as $\alpha$ denotes a multiindex $\alpha=\left(\alpha_{1}, \alpha_{2}\right) \in \mathbb{Z}_{+}^{2}$. For $\alpha=\left(\alpha_{1}, \alpha_{2}\right) \in \mathbb{Z}_{+}^{2}$ and $\mathbf{x}=\left(x_{1}, x_{2}\right) \in \mathbb{R}^{2}, \mathbf{x}^{\alpha}$ denotes the monomial $x_{1}^{\alpha_{1}} x_{2}^{\alpha_{2}}$ of $\mathbf{x}$ of degree $|\alpha|:=\alpha_{1}+\alpha_{2}$. In the following $\mathbf{u}_{\mathbf{n}}^{\alpha}, \widetilde{\mathbf{u}}_{\mathbf{n}}^{\alpha}$ are some $1 \times r$ vectors.

Let $S(\mathbf{x})$ and $T(\mathbf{x})$ be the associated function vectors. Then both $S(\mathbf{x})$ and $T(\mathbf{x})$ generate polynomials of total degree up to $m$ :

$$
\sum_{\mathbf{n} \in \mathbf{Z}^{2}} \mathbf{u}_{\mathbf{n}}^{\alpha} S(\mathbf{x}-\mathbf{n})=\mathbf{x}^{\alpha}, \mathbf{x} \in \mathbb{R}^{2},|\alpha| \leq m
$$

and

$$
\sum_{\mathbf{n} \in \mathbf{Z}^{2}} \widetilde{\mathbf{u}}_{\mathbf{n}}^{\alpha} T(\mathbf{x}-\widetilde{\mathbf{n}})=\mathbf{x}^{\alpha}, \mathbf{x} \in \mathbb{R}^{2},|\alpha| \leq m,
$$

with $1 \times r$ row vectors $\mathbf{u}_{\mathbf{n}}^{\alpha}, \widetilde{\mathbf{u}}_{\mathbf{n}}^{\alpha}$ satisfying

$$
\sum_{\mathbf{k} \in \mathbf{Z}^{2}} \mathbf{u}_{\mathbf{k}}^{\alpha} Q_{\mathbf{n}-2 \mathbf{k}}=\frac{1}{2^{|\alpha|}} \mathbf{u}_{\mathbf{n}}^{\alpha}, \sum_{\mathbf{k} \in \mathbf{Z}^{2}} \widetilde{\mathbf{u}}_{\mathbf{k}}^{\alpha} P_{\mathbf{n}-2 \mathbf{k}}=\frac{1}{2^{|\alpha|}} \widetilde{\mathbf{u}}_{\mathbf{n}}^{\alpha}, \mathbf{n} \in \mathbb{Z}^{2} .
$$

Next, we obtain conditions for $H_{k}, \mathbf{a}_{\mathbf{k}}, \mathbf{b}_{\mathbf{k}}, \mathbf{d}_{\mathbf{k}}$ such that $V_{0}$ defined by (2.9) reproduces polynomials of total degree $m$, namely, there are vectors 
$Y^{\alpha}(k),|\alpha| \leq m$ such that

$\sum_{k \in \mathbf{Z}} Y^{\alpha}(k) \Phi(\mathbf{x}-(0, k))+\sum_{\mathbf{n} \in \Gamma_{1}} \mathbf{u}_{\mathbf{n}}^{\alpha} S(\mathbf{x}-\mathbf{n})+\sum_{\mathbf{m} \in \Gamma_{2}} \widetilde{\mathbf{u}}_{\mathbf{m}}^{\alpha} T(\mathbf{x}-\widetilde{\mathbf{m}})=\mathbf{x}^{\alpha}, \mathbf{x} \in \mathbb{R}^{2}$,

which is equivalent to that for $|\alpha| \leq m$, and for $\mathbf{x}=(x, y) \in \mathbb{R}^{2}$ with $x \leq 0$

$$
\sum_{k \in \mathbf{Z}} Y^{\alpha}(k) \Phi(\mathbf{x}-(0, k))+\sum_{\mathbf{n} \in \Gamma_{1}} \mathbf{u}_{\mathbf{n}}^{\alpha} S(\mathbf{x}-\mathbf{n})=\mathbf{x}^{\alpha},
$$

and for $\mathbf{x}=(x, y) \in \mathbb{R}^{2}$ with $x>0$,

$$
\sum_{k \in \mathbf{Z}} Y^{\alpha}(k) \Phi(\mathbf{x}-(0, k))+\sum_{\mathbf{m} \in \Gamma_{2}} \widetilde{\mathbf{u}}_{\mathbf{m}}^{\alpha} T(\mathbf{x}-\widetilde{\mathbf{m}})=\mathbf{x}^{\alpha} .
$$

Before giving the main result on the polynomial reproduction, we first have following lemmas which will be used later.

Lemma 1. Let $N(\mathbf{x})$ be the function vector in (2.13) and $\mathbf{u}_{\mathbf{n}}^{\alpha},|\alpha| \leq m$, be the row vectors satisfying (3.1). If there are (row) vectors $Y^{\alpha}(k)=$ $\left[Y_{1}^{\alpha}(k), Y_{2}^{\alpha}(k), Y_{3}^{\alpha}(k)\right]$ such that for $\mathbf{n} \in \Gamma_{1}$,

$$
\sum_{k \in \mathbf{Z}}\left\{Y_{1}^{\alpha}(k) a_{\mathbf{n}-(0,2 k)}+Y_{2}^{\alpha}(k) b_{\mathbf{n}-(0,2 k)}\right\}+\sum_{\mathbf{n}^{\prime} \in \Gamma_{1}} \mathbf{u}_{\mathbf{n}^{\prime}}^{\alpha} Q_{\mathbf{n}-2 \mathbf{n}^{\prime}}=\frac{1}{2^{|\alpha|}} \mathbf{u}_{\mathbf{n}}^{\alpha},
$$

then for $\mathbf{x}=(x, y) \in \mathbb{R}^{2}, x \leq 0$,

$$
\sum_{k \in \mathbf{Z}} Y^{\alpha}(k) N(x, y-k)+\sum_{\mathbf{n} \in \Gamma_{1}} \mathbf{u}_{\mathbf{n}}^{\alpha} S(\mathbf{x}-\mathbf{n})=\frac{1}{2^{|\alpha|}} \sum_{\mathbf{n} \in \Gamma_{1}} \mathbf{u}_{\mathbf{n}}^{\alpha} S(2 \mathbf{x}-\mathbf{n}) .
$$

Lemma 2. Let $N(\mathbf{x})$ be the function vector in (2.13) and $\widetilde{\mathbf{u}}_{\mathbf{n}}^{\alpha},|\alpha| \leq m$, be the row vectors satisfying (3.2). If there are (row) vectors $Y^{\alpha}(k)=$ $\left[Y_{1}^{\alpha}(k), Y_{2}^{\alpha}(k), Y_{3}^{\alpha}(k)\right]$ such that for $\mathbf{m} \in \Gamma_{2}$,

$$
\sum_{k \in \mathbf{Z}}\left\{Y_{1}^{\alpha}(k) \mathbf{a}_{\mathbf{m}-(0,2 k)}+Y_{3}^{\alpha}(k) \mathbf{d}_{\mathbf{m}-(0,2 k)}\right\}+\sum_{\mathbf{m}^{\prime} \in \Gamma_{2}} \widetilde{\mathbf{u}}_{\mathbf{m}^{\prime}}^{\alpha} P_{\mathbf{m}-2 \widetilde{\mathbf{m}}^{\prime}}=\frac{1}{2^{|\alpha|}} \widetilde{\mathbf{u}}_{\mathbf{m}}^{\alpha},
$$

then for $\mathbf{x}=(x, y) \in \mathbb{R}^{2}, x>0$,

$$
\sum_{k \in \mathbf{Z}} Y^{\alpha}(k) N(x, y-k)+\sum_{\mathbf{m} \in \Gamma_{2}} \widetilde{\mathbf{u}}_{\mathbf{m}}^{\alpha} T(\mathbf{x}-\widetilde{\mathbf{m}})=\frac{1}{2^{|\alpha|}} \sum_{\mathbf{m} \in \Gamma_{2}} \widetilde{\mathbf{u}}_{\mathbf{m}}^{\alpha} T(2 \mathbf{x}-\widetilde{\mathbf{m}}) .
$$


One can obtain Lemma 1 and Lemma 2 by direct calculations.

Recall that for $G(\omega)=\frac{1}{2} \sum_{k \in \mathbf{Z}} G_{k} e^{-i k \omega}$, where $i=\sqrt{-1}$, and $\left\{G_{k}\right\}_{k}$ is a 1-D matrix-valued mask with finitely many $s \times s$ matrices $G_{k}$ nonzero, we say $G(\omega)$ has sum rule of order $m+1$ if there exist $1 \times s$ row constant vectors $\mathbf{y}_{0}, \mathbf{y}_{1}, \cdots, \mathbf{y}_{m}$ with $\mathbf{y}_{0} \neq[0, \cdots, 0]$ such that for $j=0, \cdots, m$,

$$
\sum_{n=0}^{j}\left(\begin{array}{c}
j \\
n
\end{array}\right) 2^{n} i^{j-n} \mathbf{y}_{n}\left(D^{j-n} G\right)(0)=\mathbf{y}_{j}, \sum_{n=0}^{j}\left(\begin{array}{c}
j \\
n
\end{array}\right) 2^{n} i^{j-n} \mathbf{y}_{n}\left(D^{j-n} G\right)(\pi)=0
$$

where $D^{j-n} G(\omega)$ denotes the $(j-n)$-th derivative of $G(\omega)$.

Let $\left\{H_{k}\right\}_{k}$ be the 1-D matrix-valued mask for the quad/triangle scheme. Suppose that $H(\omega):=\frac{1}{2} \sum_{k \in \mathbf{Z}} H_{k} e^{-i k \omega}$ has sum rule of order $m+1$ with the vectors $\mathbf{y}_{0}^{0}, \mathbf{y}_{1}^{0}, \cdots, \mathbf{y}_{m}^{0}$. Furthermore, we assume for each $j$ with $1 \leq j \leq m$, $2^{j} H(\omega)$ has sum rule of order $m+1-j$ with the vectors $\mathbf{y}_{0}^{j}, \mathbf{y}_{1}^{j}, \cdots, \mathbf{y}_{m-j}^{j}$. Define for $k \in \mathbb{Z}$,

$$
\begin{aligned}
& Y^{0, l}(k)=\sum_{n=0}^{l}\left(\begin{array}{l}
l \\
n
\end{array}\right) k^{l-n} \mathbf{y}_{n}^{0}, l=0,1, \cdots, m, \\
& Y^{1, l}(k)=\sum_{n=0}^{l}\left(\begin{array}{l}
l \\
n
\end{array}\right) k^{l-n} \mathbf{y}_{n}^{1}, l=0,1, \cdots, m-1, \\
& \vdots \\
& Y^{m-1, l}(k)=\sum_{n=0}^{l}\left(\begin{array}{l}
l \\
n
\end{array}\right) k^{l-n} \mathbf{y}_{n}^{m-1}, l=0,1, \\
& Y^{m, 0}(k)=\mathbf{y}_{0}^{m} .
\end{aligned}
$$

Lemma 3. Assume that for $0 \leq j \leq m, 2^{j} H(\omega)$ has sum rule of order $m+1-j$ with vectors $\mathbf{y}_{0}^{j}, \mathbf{y}_{1}^{j}, \cdots, \mathbf{y}_{m-j}^{j}$. Let $Y^{\alpha}(k)$ be the vectors defined above. Then

$$
\sum_{k^{\prime} \in \mathbf{Z}} Y^{\alpha}\left(k^{\prime}\right) H_{k-2 k^{\prime}}=\frac{1}{2^{|\alpha|}} Y^{\alpha}(k), k \in \mathbb{Z},|\alpha| \leq m, \alpha \in \mathbb{Z}_{+}^{2} .
$$

Since for each $j$ with $0 \leq j \leq m, 2^{j} H(\omega)$ has sum rule of order $m+1-j$ with vectors $\mathbf{y}_{0}^{j}, \mathbf{y}_{1}^{j}, \cdots, \mathbf{y}_{m-j}^{j}$, one has that (see Theorem 3 in [5]) for $l=$ 


$$
\begin{aligned}
0, \cdots, m-j, Y^{j, l}(k)= & \sum_{n=0}^{l}\left(\begin{array}{c}
l \\
n
\end{array}\right) k^{l-n} \mathbf{y}_{n}^{j} \text { satisfies } \\
& \sum_{k^{\prime} \in \mathbf{Z}} Y^{j, l}\left(k^{\prime}\right) 2^{j} H_{k-2 k^{\prime}}=\frac{1}{2^{l}} Y^{j, l}(k) .
\end{aligned}
$$

Therefore, $\sum_{k^{\prime} \in \mathbf{Z}} Y^{j, l}\left(k^{\prime}\right) H_{k-2 k^{\prime}}=\frac{1}{2^{j+l}} Y^{j, l}(k)$, which is (3.10).

Now we have our theorem on the polynomial reproduction of a quad/triangle scheme.

Theorem 1. For a quad/triangle scheme with $\left\{H_{k}\right\}_{k},\left\{Q_{\mathbf{k}}\right\}_{\mathbf{k}},\left\{P_{\mathbf{k}}\right\}_{\mathbf{k}}$ and $\mathbf{a}_{\mathbf{k}}, \mathbf{b}_{\mathbf{k}}, \mathbf{d}_{\mathbf{k}}$. Assume both $\left\{Q_{\mathbf{k}}\right\}_{\mathbf{k}}$ and $\left\{P_{\mathbf{k}}\right\}_{\mathbf{k}}$ have sum rule of order $m+1$ with vectors $\mathbf{u}^{\alpha}, \widetilde{\mathbf{u}}^{\alpha},|\alpha| \leq m$, resp. Suppose for $0 \leq j \leq m, 2^{j} H(\omega)$ has sum rule of order $m+1-j$ with vectors $\mathbf{y}_{0}^{j}, \mathbf{y}_{1}^{j}, \cdots, \mathbf{y}_{m-j}^{j}$. Let $\mathbf{u}_{\mathbf{k}}^{\alpha}$, $\widetilde{\mathbf{u}}_{\mathbf{k}}^{\alpha}$ and $Y^{\alpha}(k)$ be the vectors defined above. If (3.6) and (3.8) hold, then $V_{0}$ defined by (2.9) reproduces polynomials of total degree up to $\mathrm{m}$ :

$\sum_{k \in \mathbf{Z}} Y^{\alpha}(k) \Phi(\mathbf{x}-(0, k))+\sum_{\mathbf{n} \in \Gamma_{1}} \mathbf{u}_{\mathbf{n}}^{\alpha} S(\mathbf{x}-\mathbf{n})+\sum_{\mathbf{m} \in \Gamma_{2}} \widetilde{\mathbf{u}}_{\mathbf{m}}^{\alpha} T(\mathbf{x}-\widetilde{\mathbf{m}})=\mathbf{x}^{\alpha},|\alpha| \leq m$.

Remark 1. Because only finitely many of $\mathbf{a}_{\mathbf{n}}, \mathbf{b}_{\mathbf{n}}, \mathbf{d}_{\mathbf{n}}$ are nonzero, for $\mathbf{n}=$ $\left(n_{1}, n_{2}\right) \in \Gamma_{1}, \mathbf{m}=\left(m_{1}, m_{2}\right) \in \Gamma_{2}$ with $-n_{1}, m_{1}$ large enough, (3.6) and (3.8) hold automatically since in the case they are reduced to (3.3). Therefore, we need only to verify (3.6) and (3.8) for $\mathbf{n}, \mathbf{m}$ near the $y$-axis.

To prove Theorem 1, we need the following lemma on the structure of $Y^{\alpha}(k)$. Such a property of $Y^{\alpha}(k)$ can be found in the literature on sum rule order of matrix-valued masks. Here we provide its proof for the selfcontaining purpose.

Lemma 4. For $j=0, \cdots, m$,

$$
Y^{j, l}(k+1)=\sum_{n=0}^{l}\left(\begin{array}{l}
l \\
n
\end{array}\right) Y^{j, n}(k) .
$$

Proof. By the definitions of $Y^{j, l}(k)$, it is easy to get

$$
\begin{aligned}
Y^{j, l}(k+1) & =\sum_{n=0}^{l}\left(\begin{array}{l}
l \\
n
\end{array}\right)(k+1)^{l-n} \mathbf{y}_{n}^{j}=\sum_{n=0}^{l}\left(\begin{array}{l}
l \\
n
\end{array}\right) \sum_{s=0}^{l-n}\left(\begin{array}{c}
l-n \\
s
\end{array}\right) k^{l-n-s} \mathbf{y}_{n}^{j} \\
& =\sum_{s=0}^{l}\left(\begin{array}{l}
l \\
s
\end{array}\right) \sum_{n=0}^{s}\left(\begin{array}{c}
s \\
n
\end{array}\right) k^{s-n} \mathbf{y}_{n}^{j}=\sum_{s=0}^{l}\left(\begin{array}{l}
l \\
s
\end{array}\right) Y^{j, s}(k) .
\end{aligned}
$$


Proof of Theorem 1. For $|\alpha| \leq m$, let

$$
F_{\alpha}(\mathbf{x})=\sum_{k \in \mathbf{Z}} Y^{\alpha}(k) \Phi(\mathbf{x}-(0, k))+\sum_{\mathbf{n} \in \Gamma_{1}} \mathbf{u}_{\mathbf{n}}^{\alpha} S(\mathbf{x}-\mathbf{n})+\sum_{\mathbf{m} \in \Gamma_{2}} \widetilde{\mathbf{u}}_{\mathbf{m}}^{\alpha} T(\mathbf{x}-\widetilde{\mathbf{m}}) .
$$

Since for $\mathbf{x}=(x, y) \in \mathbb{R}^{2}, \sum_{\mathbf{n} \in \Gamma_{1}} \mathbf{u}_{\mathbf{n}}^{\alpha} S(\mathbf{x}-\mathbf{n})=0$ for $x \geq 0$ and $\sum_{\mathbf{m} \in \Gamma_{2}} \widetilde{\mathbf{u}}_{\mathbf{m}}^{\alpha} T(\mathbf{x}-$ $\widetilde{\mathbf{m}})=0$ for $x \leq 0,(3.7)$ and (3.9) in Lemmas 1 and 2 can be expressed as

$$
\begin{aligned}
& \sum_{k \in \mathbf{Z}} Y^{\alpha}(k) N(\mathbf{x}-(0, k))+\sum_{\mathbf{n} \in \Gamma_{1}} \mathbf{u}_{\mathbf{n}}^{\alpha} S(\mathbf{x}-\mathbf{n})+\sum_{\mathbf{m} \in \Gamma_{2}} \widetilde{\mathbf{u}}_{\mathbf{m}}^{\alpha} T(\mathbf{x}-\widetilde{\mathbf{m}}) \\
& =\frac{1}{2^{|\alpha|}}\left\{\sum_{\mathbf{n} \in \Gamma_{1}} \mathbf{u}_{\mathbf{n}}^{\alpha} S(2 \mathbf{x}-\mathbf{n})+\sum_{\mathbf{m} \in \Gamma_{2}} \widetilde{\mathbf{u}}_{\mathbf{m}}^{\alpha} T(2 \mathbf{x}-\widetilde{\mathbf{m}})\right\} .
\end{aligned}
$$

This, together with the nonhomogeneous refinement equation (2.13) and Equation (3.10), leads to that

$$
\begin{aligned}
& F_{\alpha}(\mathbf{x})=\sum_{k \in \mathbf{Z}} Y^{\alpha}(k)\left\{\sum_{k^{\prime} \in \mathbf{Z}} H_{k^{\prime}-2 k} \Phi\left(2 \mathbf{x}-\left(0, k^{\prime}\right)\right)+N(\mathbf{x}-(0, k))\right\} \\
& \quad+\sum_{\mathbf{n} \in \Gamma_{1}} \mathbf{u}_{\mathbf{n}}^{\alpha} S(\mathbf{x}-\mathbf{n})+\sum_{\mathbf{m} \in \Gamma_{2}} \widetilde{\mathbf{u}}_{\mathbf{m}}^{\alpha} T(\mathbf{x}-\widetilde{\mathbf{m}}) \\
& =\sum_{k^{\prime} \in \mathbf{Z}}\left\{\sum_{k \in \mathbf{Z}} Y^{\alpha}(k) H_{k^{\prime}-2 k} \Phi\left(2 \mathbf{x}-\left(0, k^{\prime}\right)\right)\right\} \\
& \quad+\sum_{k \in \mathbf{Z}} Y^{\alpha}(k) N(\mathbf{x}-(0, k))+\sum_{\mathbf{n} \in \Gamma_{1}} \mathbf{u}_{\mathbf{n}}^{\alpha} S(\mathbf{x}-\mathbf{n})+\sum_{\mathbf{m} \in \Gamma_{2}} \widetilde{\mathbf{u}}_{\mathbf{m}}^{\alpha} T(\mathbf{x}-\widetilde{\mathbf{m}}) \\
& =\sum_{k^{\prime} \in \mathbf{Z}} \frac{1}{2^{|\alpha|}} Y^{\alpha}\left(k^{\prime}\right) \Phi\left(2 \mathbf{x}-\left(0, k^{\prime}\right)\right)+\frac{1}{2^{|\alpha|}}\left\{\sum_{\mathbf{n} \in \Gamma_{1}} \mathbf{u}_{\mathbf{n}}^{\alpha} S(2 \mathbf{x}-\mathbf{n})+\sum_{\mathbf{m} \in \Gamma_{2}} \widetilde{\mathbf{u}}_{\mathbf{m}}^{\alpha} T(2 \mathbf{x}-\widetilde{\mathbf{m}})\right\} \\
& =\frac{1}{2^{|\alpha|}} F_{\alpha}(2 \mathbf{x}) .
\end{aligned}
$$

Since $\operatorname{supp}(\Phi) \subset[-3,3] \times(-\infty, \infty)$, for $\mathbf{x}=(x, y)$ with $x<-3, F_{\alpha}(\mathbf{x})=$ $\sum_{\mathbf{n} \in \Gamma_{1}} \mathbf{u}_{\mathbf{n}}^{\alpha} S(\mathbf{x}-\mathbf{n})=\mathbf{x}^{\alpha}$ because of (3.1). Thus for $\mathbf{x}=(x, y)$ with $x<-\frac{3}{2}$,

$$
F_{\alpha}(\mathbf{x})=\frac{1}{2^{|\alpha|}} F_{\alpha}(2 \mathbf{x})=\frac{1}{2^{|\alpha|}}(2 \mathbf{x})^{\alpha}=\mathbf{x}^{\alpha}
$$

More general, by the relation $F_{\alpha}(\mathbf{x})=\frac{1}{2^{n|\alpha|}} F_{\alpha}\left(2^{n} \mathbf{x}\right)$, we have that $F_{\alpha}(\mathbf{x})=\mathbf{x}^{\alpha}$ for $\mathbf{x}=(x, y)$ with $x<-\frac{3}{2^{n}}, n \in \mathbb{Z}_{+}$. Therefore, $F_{\alpha}(\mathbf{x})=\mathbf{x}^{\alpha}$ for $\mathbf{x}=(x, y)$ with $x<0$. 
One can show similarly that $F_{\alpha}(\mathbf{x})=\mathbf{x}^{\alpha}$ for $\mathbf{x}=(x, y)$ with $x>0$. Next, we show that $F_{\alpha}(\mathbf{x})=\mathbf{x}^{\alpha},|\alpha| \leq m$ holds for $\mathbf{x}=(x, y)$ with $x=0$, namely, $F_{\left(0, \alpha_{2}\right)}(0, y)=y^{\alpha_{2}}$ and $F_{\left(\alpha_{1}, \alpha_{2}\right)}(0, y)=0$ for $\alpha_{1}>0$.

For $\mathbf{x}=(x, y)$ with $x=0, F_{\alpha}(\mathbf{x})$ is given by

$$
F_{\alpha}(0, y)=\sum_{k \in \mathbf{Z}} Y^{\alpha}(k) \Phi(0, y-k) .
$$

Notice that $\Phi(0, y)$ is the solution $\phi(y)$ of the homogeneous equation

$$
\phi(y)=\sum_{k \in \mathbf{Z}} H_{k} \phi(2 y-k) .
$$

Since $H(\omega)$ has sum rule of order $m+1$ with vectors $\mathbf{y}_{0}^{0}, \mathbf{y}_{1}^{0}, \cdots, \mathbf{y}_{m}^{0}, \boldsymbol{\phi}$ and its integer-shifts $\phi(y-k)$ reproduce polynomials $y^{\alpha_{2}}, 0 \leq \alpha_{2} \leq m$ with (vector) coefficients $Y^{\left(0, \alpha_{2}\right)}(k)$, namely, $\sum_{k \in \mathbf{Z}} Y^{\left(0, \alpha_{2}\right)}(k) \phi(y-k)=y^{\alpha_{2}}$, see $[5,14,7,6]$. Therefore, $F_{\left(0, \alpha_{2}\right)}(0, y)=y^{\alpha_{2}}, 0 \leq \alpha_{2} \leq m$, as desired.

Next, we prove that $F_{\left(\alpha_{1}, \alpha_{2}\right)}(0, y)=0$ when $\alpha_{1}>0$. To this regard, we denote $S_{l}^{j}(y):=F_{(j, l)}(0, y)=\sum_{k \in \mathbf{Z}} Y^{j, l}(k) \Phi(0, y-k)$. What we need to prove is for each $j$ with $1 \leq j \leq m, S_{l}^{j}(y)=0$ for $l=0, \cdots, m-j$. We will prove this result by induction on $l$.

For $l=0,2^{j} H(\omega)$ has sum rule of order $m+1-j$ with vectors $\mathbf{y}_{0}^{j}, \cdots, \mathbf{y}_{m-j}^{j}$. By the definition of $Y^{j, l}, Y^{j, 0}(k)=\mathbf{y}_{0}^{j}$. Thus $S_{0}^{j}(y)=\sum_{k \in \mathbf{Z}} \mathbf{y}_{0}^{j} \Phi(0, y-k)$, and hence,

$$
S_{0}^{j}(y)=S_{0}^{j}(y+1) .
$$

Then the Birkhoff Ergodic Theorem implies that there is a constant $C$ such that

$$
\frac{1}{n} \sum_{k=0}^{n-1} S_{0}^{j}\left(2^{k} y\right) \longrightarrow C, \text { a.e. } n \rightarrow \infty .
$$

On the other hand, (3.10) for $\alpha=(j, 0)$ implies

$$
\begin{aligned}
S_{0}^{j}(y) & =\sum_{k \in \mathbf{Z}} Y^{j, 0}(k) \Phi(0, y-k)=\sum_{k \in \mathbf{Z}} Y^{j, 0}(k) \sum_{m \in \mathbf{Z}} H_{m} \Phi(0,2 y-2 k-m) \\
& =\sum_{m^{\prime} \in \mathbf{Z}} \sum_{k \in \mathbf{Z}} Y^{j, 0}(k) H_{m^{\prime}-2 k} \Phi\left(0,2 y-m^{\prime}\right)=\sum_{m^{\prime} \in \mathbf{Z}} 2^{-j} Y^{j, 0} \Phi\left(0,2 y-m^{\prime}\right) \\
& =2^{-j} S_{0}^{j}(2 y) .
\end{aligned}
$$


Therefore,

$$
\frac{1}{n} \sum_{k=0}^{n-1} S_{0}^{j}\left(2^{k} y\right)=\frac{2^{j n}-1}{n\left(2^{j}-1\right)} S_{0}^{j}(y) .
$$

This, together with (3.12) and the fact $\frac{2^{j n}-1}{n\left(2^{j}-1\right)} \rightarrow \infty$ as $n \rightarrow \infty$, leads to that $S_{0}^{j}(y)=0$.

Next, we show $S_{l}^{j}(y)=0$ under the induction hypothesis that $S_{l^{\prime}}^{j}(y)=0$ for $l^{\prime}=0,1, \cdots, l-1$. By $(3.11)$, we have

$$
\begin{aligned}
& S_{l}^{j}(y+1)=\sum_{k \in \mathbf{Z}} Y^{j, l}(k) \Phi(0, y-k+1)=\sum_{k \in \mathbf{Z}} Y^{j, l}(k+1) \Phi(0, y-k) \\
& =\sum_{k \in \mathbf{Z}} \sum_{n=0}^{l}\left(\begin{array}{l}
l \\
n
\end{array}\right) Y^{j, n}(k) \Phi(0, y-k)=\sum_{n=0}^{l}\left(\begin{array}{l}
l \\
n
\end{array}\right) \sum_{k \in \mathbf{Z}} Y^{j, n}(k) \Phi(0, y-k) \\
& =\sum_{n=0}^{l}\left(\begin{array}{l}
l \\
n
\end{array}\right) S_{n}^{j}(y)=S_{l}^{j}(y) .
\end{aligned}
$$

While, by (3.10), we have

$$
\begin{aligned}
& S_{l}^{j}(y)=\sum_{k \in \mathbf{Z}} Y^{j, l}(k) \Phi(0, y-k)=\sum_{k \in \mathbf{Z}} Y^{j, l}(k) \sum_{s \in \mathbf{Z}} H_{s-2 k} \Phi(0,2 y-s) \\
& =\frac{1}{2^{j+l}} \sum_{s \in \mathbf{Z}} Y^{j, l}(s) \Phi(0,2 y-s)=\frac{1}{2^{j+l}} S_{l}^{j}(2 y) .
\end{aligned}
$$

Thus, the Birkhoff Ergodic Theorem again implies $S_{l}^{j}(y)=0$, namely, $F_{j, l}(0, y)=0$. Therefore, $F_{\alpha}(\mathbf{x})=\mathbf{x}^{\alpha},|\alpha| \leq m$ also holds for $\mathbf{x}=(0, y)$.

Next, let us look at the approximation reproduction of Stam-Loop's and Levin-Levin's schemes. For these two schemes, $S(\mathbf{x})$ and $T(\mathbf{x})$ are resp. the bi-cubic spline and the $C^{2}$ quartic box-spline $B_{222}$ along the triangle mesh on the left of Fig.2. Each of $S(\mathbf{x})$ and $T(\mathbf{x})$ reproduces polynomials of total degree up to at least 2. More precisely,

$$
\sum_{\mathbf{k} \in \mathbf{Z}^{2}} u_{\mathbf{k}}^{\alpha} S(\mathbf{x}-\mathbf{k})=\mathbf{x}^{\alpha}, \sum_{\mathbf{k} \in \mathbf{Z}^{2}} \widetilde{u}_{\mathbf{k}}^{\alpha} T(\mathbf{x}-\widetilde{\mathbf{k}})=\mathbf{x}^{\alpha}, \mathbf{x} \in \mathbb{R}^{2},|\alpha| \leq 2,
$$

where

$$
\left\{\begin{array}{l}
u_{\mathbf{k}}^{0,0}=1, u_{\mathbf{k}}^{1,0}=k_{1}, u_{\mathbf{k}}^{0,1}=k_{2}, \\
u_{\mathbf{k}}^{2,0}=k_{1}^{2}-\frac{1}{3}, u_{\mathbf{k}}^{1,1}=k_{1} k_{2}, u_{\mathbf{k}}^{0,2}=k_{2}^{2}-\frac{1}{3},
\end{array}\right.
$$


and

$$
\left\{\begin{array}{l}
\widetilde{u}_{\mathbf{k}}^{0,0}=1, \widetilde{u}_{\mathbf{k}}^{1,0}=k_{1}, \widetilde{u}_{2 k_{1}, k_{2}}^{0,1}=k_{2}, \widetilde{u}_{2 k_{1}+1, k_{2}}^{0,1}=k_{2}-\frac{1}{2}, \\
\widetilde{u}_{\mathbf{k}}^{2,0}=k_{1}^{2}-\frac{1}{3}, \widetilde{u}_{2 k_{1}, k_{2}}^{1,1}=2 k_{1} k_{2}, \widetilde{u}_{2 k_{1}+1, k_{2}}^{1,1}=\left(2 k_{1}+1\right)\left(k_{2}-\frac{1}{2}\right), \\
\widetilde{u}_{2 k_{1}, k_{2}}^{0,2}=k_{2}^{2}-\frac{1}{4}, \widetilde{u}_{2 k_{1}+1, k_{2}}^{0,2}=\left(k_{2}-\frac{1}{2}\right)^{2}-\frac{1}{4} .
\end{array}\right.
$$

For Stam-Loop's scheme, one can verify that $H(\omega)=\frac{1}{2} \sum_{k=-2}^{2} H_{k} e^{-i k \omega}$ (see [8] for $H_{k}$ ) has sum rule of order 2 with vectors

$$
\mathbf{y}_{0}^{0}=[1,1,1], \mathbf{y}_{1}^{0}=\left[0,0,-\frac{1}{2}\right]
$$

and that $2 H(\omega)$ has sum rule of order 2 with the vectors

$$
\mathbf{y}_{1}^{0}=[0,-1,1]
$$

With $u_{\mathbf{n}}, \widetilde{u}_{\mathbf{m}}$ given in (3.13) and (3.14) resp., one can verify (by tedious calculation) that (3.6) and (3.8) hold for $|\alpha| \leq 1$. Thus Stam-Loop's scheme can reproduce $1, x, y$.

For Levin-Levin's scheme, one can show that $H(\omega)=\frac{1}{2} \sum_{k=-3}^{4} H_{k} e^{-i k \omega}$ (see [8] again for $H_{k}$ ) has sum rule of of order 3 with the vectors

$$
\mathbf{y}_{0}^{0}=[1,1,1], \mathbf{y}_{1}^{0}=\left[0,0,-\frac{1}{2}\right], \mathbf{y}_{2}^{0}=\left[-\frac{1}{3},-\frac{1}{3}, 0\right] ;
$$

$2 H(\omega)$ has sum rule of order 2 with the vectors

$$
\mathbf{y}_{0}^{1}=[0,-1,1], \mathbf{y}_{1}^{1}=\left[0,0,-\frac{1}{2}\right]
$$

and that $4 H(\omega)$ has sum rule of order 1 with the vector

$$
\mathbf{y}_{0}^{2}=\left[-\frac{1}{3}, \frac{2}{3}, \frac{2}{3}\right]
$$

With $u_{\mathbf{n}}, \widetilde{u}_{\mathbf{m}}$ given in (3.13) and (3.14) resp., one can obtain that (3.6) and (3.8) hold for $|\alpha| \leq 2$. Thus Levin-Levin's scheme can reproduce constant, linear and quadratic polynomials. 

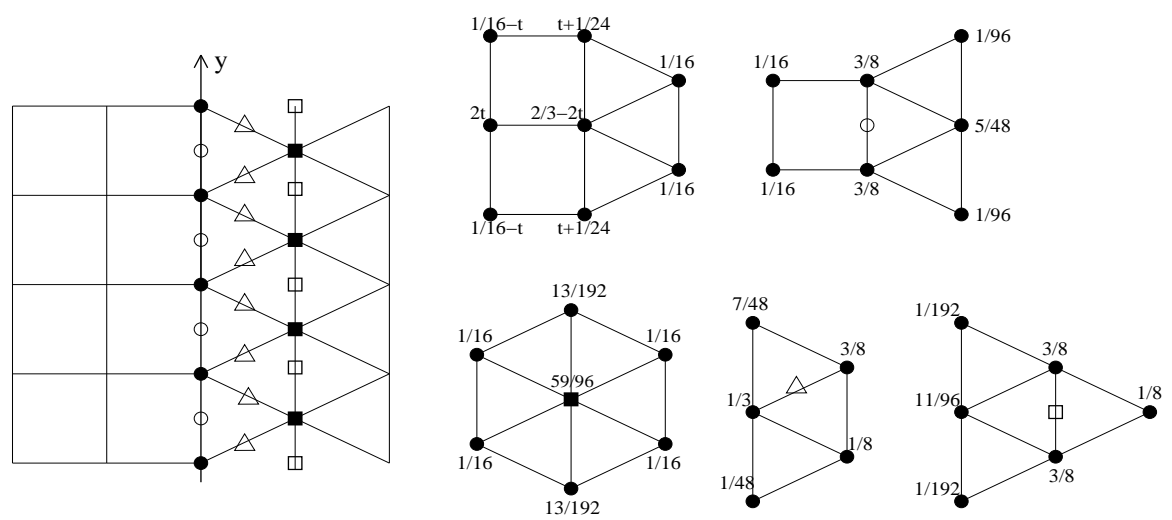

Figure 7: Templates of a new scheme for "even" vertices (top-left) and "odd" vertices (top-right) on the y-axis, templates for "even" vertices (bottom-left), and "odd" vertices (bottom-middle and bottom-right) on right and near the $y$-axis

\section{New quad/triangle schemes}

In this section we show that Theorem 1 leads to new schemes.

\subsection{Quad triangle scheme reproducing quadratic polynomials}

In Fig.7, we provide the templates of a quad/triangle scheme for vertices near the $y$-axis are shown in Fig.7, while Catmull-Clark's scheme for the vertices on the left of the $y$-axis is used, and Loop's scheme for the vertices on the right and little far from the $y$-axis is applied. For this scheme, the associated nonzero $H_{k}$ are

$$
\begin{aligned}
& H_{0}=\frac{1}{48}\left[\begin{array}{ccc}
32-96 t & 18 & 16 \\
96 t & 18 & 0 \\
3 & 0 & 18
\end{array}\right], H_{-1}=\frac{1}{48}\left[\begin{array}{ccc}
18 & 12 & 7 \\
3 & 12 & 0 \\
5 & 0 & 18
\end{array}\right], \\
& H_{1}=\frac{1}{96}\left[\begin{array}{ccc}
36 & 24 & 32 \\
6 & 24 & 0 \\
1 & 0 & 12
\end{array}\right], H_{-2}=\frac{1}{48}\left[\begin{array}{ccc}
48 t+2 & 3 & 1 \\
3-48 t & 3 & 0 \\
3 & 0 & 6
\end{array}\right] \\
& H_{2}=\frac{1}{48}\left[\begin{array}{ccc}
48 t+2 & 3 & 7 \\
3-48 t & 3 & 0 \\
0 & 0 & 0
\end{array}\right], H_{-3}=\frac{1}{96}\left[\begin{array}{ccc}
0 & 0 & 0 \\
0 & 0 & 0 \\
1 & 0 & 0
\end{array}\right], H_{3}=\frac{1}{48}\left[\begin{array}{ccc}
0 & 0 & 1 \\
0 & 0 & 0 \\
0 & 0 & 0
\end{array}\right]
\end{aligned}
$$


and the corresponding nonzero $a_{j, k}, b_{j, k}, d_{j, k}$ are listed as follows:

$$
\begin{aligned}
& a_{2,0}=\frac{11}{96}, a_{2,1}=a_{2,-1}=\frac{1}{16}, a_{-2,0}=\frac{3}{32}, a_{2,2}=a_{2,-2}=\frac{1}{192}, \\
& a_{-2,1}=a_{-2,-1}=\frac{1}{16}, a_{-2,2}=a_{-2,-2}=\frac{1}{64}, b_{-2,0}=\frac{9}{16}, \\
& b_{-2,1}=b_{-2,-1}=\frac{3}{8}, b_{-2,2}=b_{-2,-2}=\frac{3}{32}, b_{-3,0}=\frac{3}{8}, b_{-3,1}=b_{-3,-1}=\frac{1}{4}, \\
& b_{-3,2}=b_{-3,-2}=\frac{1}{16}, b_{-4,0}=\frac{3}{32}, b_{-4,1}=b_{-4,-1}=\frac{1}{16}, b_{-4,2}=b_{-4,-2}=\frac{1}{64}, \\
& d_{2,-1}=\frac{59}{96}, d_{2,0}=d_{2,-2}=\frac{3}{8}, d_{2,1}=d_{2,-3}=\frac{13}{192}, \\
& d_{3,-1}=d_{3,-2}=\frac{3}{8}, d_{3,0}=d_{3,-3}=\frac{1}{8}, d_{4,-1}=\frac{1}{8}, d_{4,0}=d_{4,-2}=\frac{1}{16} .
\end{aligned}
$$

With $H(\omega)=\frac{1}{2} \sum_{k \in \mathbf{Z}} H_{k} e^{-i k \omega}$, one can show that $H(\omega), 2 H(\omega)$ and $4 H(\omega)$

have sum rule of orders 3,2 and 1 resp. with vectors $\mathbf{y}_{0}^{0}, \mathbf{y}_{1}^{0}, \mathbf{y}_{2}^{0}, \mathbf{y}_{0}^{1}, \mathbf{y}_{1}^{1}$ and $\mathbf{y}_{0}^{2}$ given by (3.15), (3.16) and (3.17). We can also verify the conditions (3.6) and (3.8) hold. Thus by Theorem 1, this new scheme reproduces polynomials of total degree up to 2.

Compared with Levin-Levin's scheme, this quad/triangle scheme has two smaller sizes of templates (at bottom-left and bottom-middle of Fig.7). This scheme has a free parameter. One may choose a suitable value for $t$ such that the resulting scheme is $C^{2}$. For example, using the smoothness estimate given in [10], for the choice of $t=\frac{1}{24}$, the resulting $\Phi$ is at least in $C^{2.4460}$ after calculating the joint spectral radius $\bar{\rho}_{17}$.

\subsection{Matrix-valued interpolatory quad/triangle scheme}

In this subsection we consider matrix-valued interpolatory quad/triangle schemes. For each scheme, we use templates in Fig.3 with $R, J, K, L, M, N$ given in (2.7) for the quad vertices on the left of the $y$-axis and use those in Fig.4 with $P, B, C, D$ given in (2.8) for the triangle vertices on the right of the $y$-axis. The corresponding masks $\left\{Q_{\mathbf{k}}\right\}$ and $\left\{P_{\mathbf{k}}\right\}$ have sum rule of (at least) order 3 , and the associated refinable function vectors $S=\left[S_{0}, S_{1}\right]^{T}, T=$ $\left[T_{0}, T_{1}\right]^{T}$ reproduce polynomials of total degree up to at least 2 with

$$
\begin{aligned}
& \mathbf{u}_{j, k}^{0,0}=[1,0], \mathbf{u}_{j, k}^{1,0}=[j, 0], \mathbf{u}_{j, k}^{0,1}=[k, 0], \\
& \mathbf{u}_{j, k}^{2,0}=\left[j^{2}, 1\right], \mathbf{u}_{j, k}^{1,1}=[j k, 0], \mathbf{u}_{j, k}^{0,2}=\left[k^{2}, 1\right] ;
\end{aligned}
$$


and

$$
\begin{aligned}
& \widetilde{\mathbf{u}}_{j, k}^{0,0}=[1,0], \widetilde{\mathbf{u}}_{j, k}^{1,0}=[j, 0], \widetilde{\mathbf{u}}_{2 j, k}^{0,1}=[k, 0], \widetilde{\mathbf{u}}_{2 j+1, k}^{0,1}=\left[k-\frac{1}{2}, 0\right], \widetilde{\mathbf{u}}_{j, k}^{2,0}=\left[j^{2}, 1\right], \\
& \widetilde{\mathbf{u}}_{2 j, k}^{1,1}=[2 j k, 0], \widetilde{\mathbf{u}}_{2 j+1, k}^{1,1}=\left[(2 j+1)\left(k-\frac{1}{2}\right), 0\right], \\
& \widetilde{\mathbf{u}}_{2 j, k}^{0,2}=\left[k^{2}, \frac{3}{4}\right], \widetilde{\mathbf{u}}_{2 j+1, k}^{0,2}=\left[\left(k-\frac{1}{2}\right)^{2}, \frac{3}{4}\right] .
\end{aligned}
$$

Example 1. We use the templates in Fig. 5 for vertices on the $y$-axis, where $G, U, V, W, W_{1}, X, Y, Z$ are $2 \times 2$ matrices. For this scheme, the nonzero $H_{k}$, $\mathbf{a}_{\mathbf{k}}, \mathbf{b}_{\mathbf{k}}, \mathbf{d}_{\mathbf{k}}$ are given in (2.18) and (2.19). We can carefully choose matrices $G, U, V, W, W_{1}, X, Y, Z$ such that the conditions in Theorem 1 for $|\alpha| \leq 1$ are satisfied, and hence, we have a matrix-valued scheme which reproduces constant and linear polynomials. For example, if we choose

$$
\begin{aligned}
G & =\left[\begin{array}{cc}
1, & -4 t_{1}-2 t_{5}-2 t_{3} \\
0, & -4 t_{1}-2 t_{6}-2 t_{3}-4 t_{2}-2 t_{4}+1 / 4
\end{array}\right], V=\left[\begin{array}{cc}
0, & t_{5} \\
0, & t_{6}
\end{array}\right] \\
U & =\left[\begin{array}{cc}
0, & t_{1}+\frac{1}{2} t_{3} \\
0, & t_{2}+\frac{1}{2} t_{4}
\end{array}\right], W=\left[\begin{array}{cc}
0 & t_{1} \\
0 & t_{2}
\end{array}\right], W_{1}=\left[\begin{array}{cc}
0, & t_{3} \\
0, & t_{4}
\end{array}\right] \\
X & =\left[\begin{array}{cc}
\frac{3}{8}, & 0 \\
t_{7}, & t_{8}
\end{array}\right], Y=\left[\begin{array}{cc}
\frac{1}{16}, & 0 \\
-\frac{1}{16}-\frac{1}{2} t_{7} & -\frac{1}{2} t_{8}+\frac{1}{16}
\end{array}\right], Z=\left[\begin{array}{cc}
\frac{1}{8}, & 0 \\
-\frac{1}{8}-t_{7}, & \frac{1}{8}-t_{8}
\end{array}\right]
\end{aligned}
$$

where $t_{j} \in \mathbb{R}$, then we have an interpolatory scheme which has such a polynomial reproducing property. Indeed, for $G, U, V, W, W_{1}, X, Y, Z$ given above, $H(\omega)=\frac{1}{2} \sum_{k=-2}^{2} H_{k} e^{-i \omega k}$ with $H_{k}$ given in (2.18) has sum rule of order 3 with vectors

$$
\mathbf{y}_{0}^{0}=[1,0,1,0,1,0], \mathbf{y}_{1}^{0}=\left[0,0,0,0,-\frac{1}{2}, 0\right], \mathbf{y}_{2}^{0}=\left[0,1,0,1, \frac{1}{2}, 0\right],
$$

$2 H(\omega)$ has sum rule of order 2 with vectors

$$
\mathbf{y}_{0}^{1}=[0,0,-1,0,1,0], \mathbf{y}_{1}^{1}=\left[0,0,0,0,-\frac{1}{2}, 0\right]
$$

and $4 H(\omega)$ has sum rule of order 1 with vector

$$
\mathbf{y}_{0}^{2}=[0,1,1,1,1,1]
$$


Therefore, $Y^{\alpha}(k),|\alpha| \leq 2$ defined by

$$
\begin{aligned}
& Y^{0,0}(k)=\mathbf{y}_{0}^{0}=[1,0,1,0,1,0], Y^{0,1}(k)=k \mathbf{y}_{0}^{0}+\mathbf{y}_{1}^{0}=\left[k, 0, k, 0, k-\frac{1}{2}, 0\right], \\
& Y^{0,2}(k)=k^{2} \mathbf{y}_{0}^{0}+2 k \mathbf{y}_{1}^{0}+\mathbf{y}_{2}^{0}=\left[k^{2}, 1, k^{2}, 1, k^{2}-k+\frac{1}{2}, 1\right], \\
& Y^{1,0}(k)=\mathbf{y}_{0}^{1}=[0,0,-1,0,1,0], Y^{1,1}(k)=k \mathbf{y}_{0}^{1}+\mathbf{y}_{1}^{1}=\left[0,0,-k, 0, k-\frac{1}{2}, 0\right], \\
& Y^{2,0}(k)=\mathbf{y}_{0}^{2}=[0,1,1,1,1,1],
\end{aligned}
$$

satisfy (3.10). We can verify that the condition (3.6) holds for $|\alpha| \leq 2$, and (3.8) holds for $\alpha=(0,0),(1,0),(0,1),(2,0),(1,1)$, but it does not hold for $\alpha=(0,2)$. Thus this scheme reproduces linear polynomials.

In [8], where the smoothness of matrix-valued quad/triangle schemes is studied, we choose some particular $t_{j}$ such that the resulting scheme is $C^{1}$.
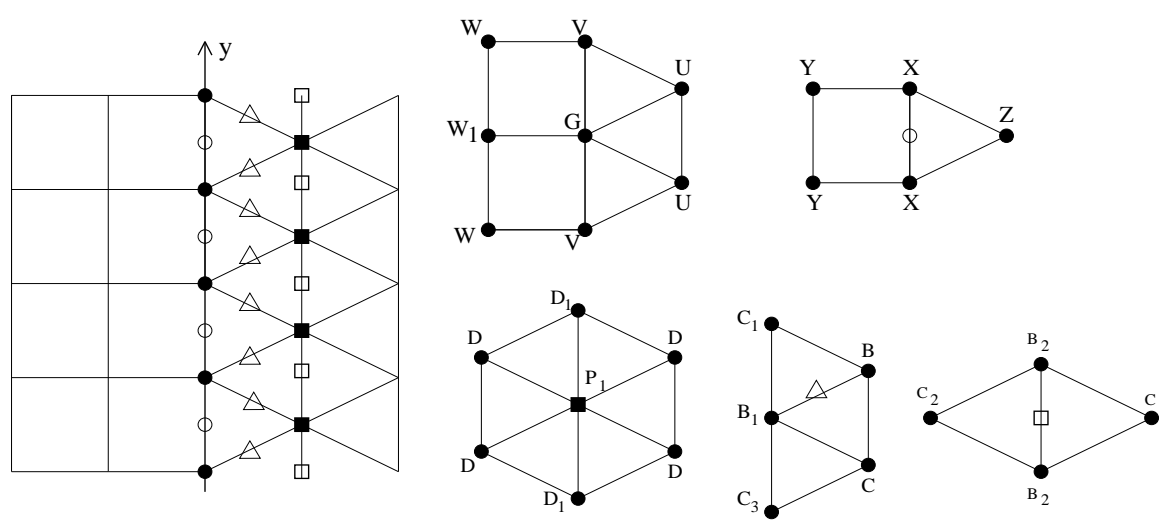

Figure 8: Templates of a new scheme for "even" vertices (top-left) and "odd" vertices (top-right) on the y-axis, templates for "even" vertices (bottom-left), and "odd" vertices (bottom-middle and bottom-right) on right and near the y-axis

Example 2. We use the templates in Fig. 8 for vertices near the $y$-axis, where

$$
\begin{aligned}
& W=\left[\begin{array}{ll}
0 & t_{1} \\
0 & t_{2}
\end{array}\right], W_{1}=\left[\begin{array}{ll}
0, & t_{3} \\
0, & t_{4}
\end{array}\right], V=\left[\begin{array}{ll}
0, & t_{5} \\
0, & t_{6}
\end{array}\right], U=\left[\begin{array}{cc}
0, & t_{1}+\frac{1}{2} t_{3} \\
0, & -3 t_{1}+4 t_{5}-\frac{7}{2} t_{3}
\end{array}\right] \\
& G=\left[\begin{array}{cc}
1, & -4 t_{1}-2 t_{5}-2 t_{3} \\
0, & 2 t_{1}-8 t_{5}+5 t_{3}-2 t_{2}-t_{4}-2 t_{6}+1 / 4
\end{array}\right], X=\left[\begin{array}{cc}
\frac{3}{8}, & -2 t_{9} \\
t_{7}, & t_{8}
\end{array}\right]
\end{aligned}
$$




$$
\begin{aligned}
& Y=\left[\begin{array}{cc}
\frac{1}{16}, & t_{9} \\
-\frac{1}{16}-t_{7} & 7 t_{9}-t_{8}+\frac{1}{8}
\end{array}\right], Z=\left[\begin{array}{cc}
\frac{1}{8}, & 2 t_{9} \\
-\frac{1}{8}, & -18 t_{9}
\end{array}\right], B_{1}=\left[\begin{array}{cc}
\frac{11}{32}, & \frac{1}{32} \\
t_{10}, & t_{11}
\end{array}\right] \\
& C_{1}=\left[\begin{array}{cc}
\frac{9}{64}, & -\frac{1}{64} \\
t_{12}, & t_{13}
\end{array}\right], C_{2}=\left[\begin{array}{cc}
\frac{1}{8}, & 0 \\
0, & 0
\end{array}\right], C_{3}=\left[\begin{array}{cc}
\frac{1}{64}, & -\frac{1}{64} \\
-\frac{1}{8}-t_{10}-t_{12}, & \frac{1}{8}-t_{11}-t_{13}
\end{array}\right] \\
& B_{2}=\left[\begin{array}{cc}
\frac{3}{8}, & 0 \\
-\frac{11}{1024}, & \frac{133}{1024}
\end{array}\right], D_{1}=\left[\begin{array}{cc}
0, & \frac{625}{2048} \\
0, & t_{14}
\end{array}\right], P_{1}=\left[\begin{array}{cc}
1, & -\frac{1785}{1024} \\
0, & -\frac{17}{32}-2 t_{14}
\end{array}\right]
\end{aligned}
$$

for $t_{j} \in \mathbb{R}$. For this scheme, the corresponding nonzero $H_{k}$ are

$$
\begin{aligned}
& H_{-2}=\left[\begin{array}{ccc}
V, & M, & C_{3} \\
W, & M, & 0 \\
U, & 0, & C
\end{array}\right], H_{-1}=\left[\begin{array}{ccc}
X, & K, & C_{1} \\
Y, & K, & 0 \\
Z, & 0, & B
\end{array}\right], H_{0}=\left[\begin{array}{ccc}
G, & J, & B_{1} \\
W_{1}, & J, & 0 \\
U, & 0, & B
\end{array}\right] \\
& H_{1}=\left[\begin{array}{ccc}
X, & K, & B_{1} \\
Y, & K, & 0 \\
0, & 0, & C
\end{array}\right], H_{2}=\left[\begin{array}{ccc}
V, & M, & C_{1} \\
W, & M, & 0 \\
0, & 0, & 0
\end{array}\right], H_{3}=\left[\begin{array}{ccc}
0, & 0, & C_{3} \\
0, & 0, & 0 \\
0, & 0, & 0
\end{array}\right]
\end{aligned}
$$

and the corresponding nonzero $\mathbf{a}_{j, k}, \mathbf{b}_{j, k}, \mathbf{d}_{j, k}$ are the same as those in Example 1 except that

$$
\mathbf{a}_{2,0}=C_{2}, \mathbf{d}_{2,0}=\mathbf{d}_{2,-2}=B_{2}, \mathbf{d}_{2,-1}=P_{1}, \mathbf{d}_{2,1}=\mathbf{d}_{2,-3}=D_{1} .
$$

For this scheme, $H(\omega)=\frac{1}{2} \sum_{k=-2}^{3} H_{k} e^{-i \omega k}$ has sum rule of orders 3 with vectors

$$
\mathbf{y}_{0}^{0}=[1,0,1,0,1,0], \mathbf{y}_{1}^{0}=\left[0,0,0,0,-\frac{1}{2}, 0\right], \mathbf{y}_{2}^{0}=\left[0,1,0,1, \frac{1}{4}, \frac{3}{4}\right],
$$

$2 H(\omega)$ has sum rule of order 2 with vectors

$$
\mathbf{y}_{0}^{1}=[0,0,-1,0,1,0], \mathbf{y}_{1}^{1}=\left[0,0,0,0,-\frac{1}{2}, 0\right]
$$

and $4 H(\omega)$ has sum rule of order 1 with vector

$$
\mathbf{y}_{0}^{2}=[0,1,1,1,1,1] \text {. }
$$

Therefore, $Y^{\alpha}(k),|\alpha| \leq 2$ defined by

$$
\begin{aligned}
& Y^{0,0}(k)=\mathbf{y}_{0}^{0}=[1,0,1,0,1,0], Y^{0,1}(k)=k \mathbf{y}_{0}^{0}+\mathbf{y}_{1}^{0}=\left[k, 0, k, 0, k-\frac{1}{2}, 0\right], \\
& Y^{0,2}(k)=k^{2} \mathbf{y}_{0}^{0}+2 k \mathbf{y}_{1}^{0}+\mathbf{y}_{2}^{0}=\left[k^{2}, 1, k^{2}, 1, k^{2}-k+\frac{1}{4}, \frac{3}{4}\right], \\
& Y^{1,0}(k)=\mathbf{y}_{0}^{1}=[0,0,-1,0,1,0], Y^{1,1}(k)=k \mathbf{y}_{0}^{1}+\mathbf{y}_{1}^{1}=\left[0,0,-k, 0, k-\frac{1}{2}, 0\right], \\
& Y^{2,0}(k)=\mathbf{y}_{0}^{2}=[0,1,1,1,1,1],
\end{aligned}
$$


satisfy (3.10). We can verify that the condition (3.6) and (3.8) holds for $|\alpha| \leq 2$. Thus, this scheme reproduces quadratic polynomials.

Based on this scheme, we construct $C^{2}$ interpolatory schemes and present some figures showing the effectiveness of the new schemes in [8].
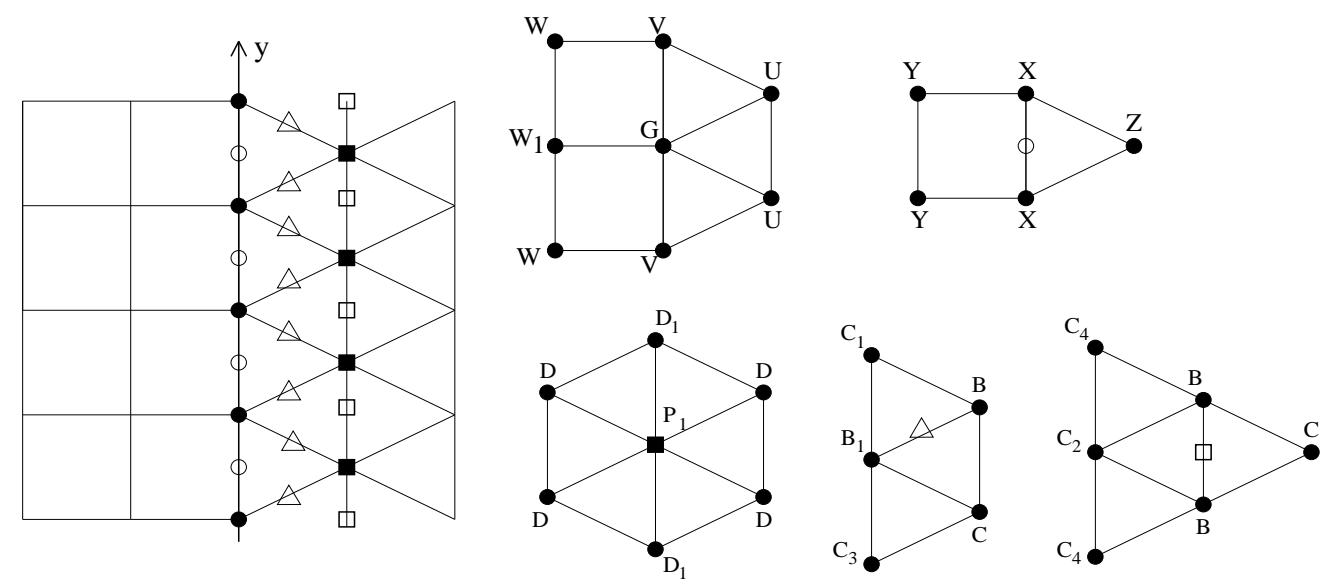

Figure 9: Templates of a new scheme for "even" vertices (top-left) and "odd" vertices (top-right) on the y-axis, templates for "even" vertices (bottom-left), and "odd" vertices (bottom-middle and bottom-right) on right and near the y-axis

Example 3. We use the templates in Fig. 9 for vertices near the y-axis, where

$$
\begin{aligned}
& W=\left[\begin{array}{cc}
0 & t_{1} \\
0 & t_{2}
\end{array}\right], W_{1}=\left[\begin{array}{cc}
0, & t_{3} \\
0, & t_{4}
\end{array}\right], V=\left[\begin{array}{cc}
0, & \frac{3}{4} t_{1}+\frac{1}{8} t_{4}+\frac{1}{4} t_{2}+\frac{7}{8} t_{3} \\
0, & t_{6}
\end{array}\right] \\
& U=\left[\begin{array}{cc}
0, & t_{1}+\frac{1}{2} t_{3} \\
0, & t_{2}+\frac{1}{2} t_{4}
\end{array}\right], G=\left[\begin{array}{cc}
1, & -\frac{11}{2} t_{1}-\frac{15}{4} t_{3}-\frac{1}{4} t_{4}-\frac{1}{2} t_{2} \\
0, & -4 t_{1}-2 t_{6}-2 t_{3}-4 t_{2}-2 t_{4}+\frac{1}{4}
\end{array}\right] \\
& X=\left[\begin{array}{cc}
\frac{3}{8}, & -2 t_{5} \\
0, & 16 t_{5}+\frac{1}{8}
\end{array}\right], Y=\left[\begin{array}{cc}
\frac{1}{16}, & t_{5} \\
-\frac{1}{16} & -9 t_{5}
\end{array}\right], Z=\left[\begin{array}{cc}
\frac{1}{8}, & 2 t_{5} \\
-\frac{1}{8}, & -18 t_{5}
\end{array}\right] \\
& B_{1}=\left[\begin{array}{cc}
\frac{11}{32}, & \frac{1}{32} \\
t_{7}, & t_{8}
\end{array}\right], C_{1}=\left[\begin{array}{cc}
\frac{9}{64}, & -\frac{1}{64} \\
-\frac{1}{2} t_{7}-\frac{307}{4096}, & -\frac{1}{2} t_{8}+\frac{241}{4096}
\end{array}\right] \\
& C_{2}=\left[\begin{array}{cc}
\frac{239}{2048}, & -\frac{5}{2048} \\
-2 t_{9}-\frac{17}{512}, & -2 t_{10}-\frac{5}{512}
\end{array}\right], C_{3}=\left[\begin{array}{cc}
\frac{1}{64}, & -\frac{1}{64} \\
-\frac{1}{2} t_{7}-\frac{205}{4096}, & \frac{271}{4096}-\frac{1}{2} t_{8}
\end{array}\right] \\
& C_{4}=\left[\begin{array}{cc}
\frac{17}{4096}, & \frac{5}{4096} \\
t_{9}, & t_{10}
\end{array}\right], D_{1}=\left[\begin{array}{cc}
0, & \frac{625}{2048} \\
0, & t_{11}
\end{array}\right], P_{1}=\left[\begin{array}{cc}
1, & -\frac{1785}{1024} \\
0, & -2 t_{11}-\frac{17}{32}
\end{array}\right]
\end{aligned}
$$


for $t_{j} \in \mathbb{R}$. For this interpolatory scheme, the corresponding nonzero $H_{k}$ are

$$
\begin{aligned}
& H_{-2}=\left[\begin{array}{ccc}
V, & M, & C_{3} \\
W, & M, & 0 \\
U, & 0, & C
\end{array}\right], H_{-1}=\left[\begin{array}{ccc}
X, & K, & C_{1} \\
Y, & K, & 0 \\
Z, & 0, & B
\end{array}\right], H_{0}=\left[\begin{array}{ccc}
G, & J, & B_{1} \\
W_{1}, & J, & 0 \\
U, & 0, & B
\end{array}\right] \\
& H_{1}=\left[\begin{array}{ccc}
X, & K, & B_{1} \\
Y, & K, & 0 \\
0, & 0, & C
\end{array}\right], H_{2}=\left[\begin{array}{ccc}
V, & M, & C_{1} \\
W, & M, & 0 \\
0, & 0, & 0
\end{array}\right], H_{3}=\left[\begin{array}{ccc}
0, & 0, & C_{3} \\
0, & 0, & 0 \\
0, & 0, & 0
\end{array}\right]
\end{aligned}
$$

and the corresponding nonzero $\mathbf{a}_{j, k}, \mathbf{b}_{j, k}, \mathbf{d}_{j, k}$ are the same as those for the Example 1 except that

$$
\mathbf{a}_{2,0}=C_{2}, \mathbf{a}_{2,2}=\mathbf{a}_{2,-2}=C_{4}, \mathbf{d}_{2,-1}=P_{1}, \mathbf{d}_{2,1}=\mathbf{d}_{2,-3}=D_{1} .
$$

For this scheme, with $H(\omega)=\frac{1}{2} \sum_{k=-2}^{3} H_{k} e^{-i \omega k}, H(\omega), 2 H(\omega)$ and $4 H(\omega)$ have sum rule of orders 3,2 and 1 resp. with vectors $\mathbf{y}_{0}^{0}, \mathbf{y}_{1}^{0}, \mathbf{y}_{2}^{0}, \mathbf{y}_{0}^{1}, \mathbf{y}_{1}^{1}$ and $\mathbf{y}_{0}^{2}$ given by (4.1), (4.2) and (4.3). We can verify that the condition (3.6) holds for $|\alpha| \leq 3$, and (3.8) holds for $\alpha=(0,0),(1,0),(0,1),(2,0),(0,2)$,

$(1,1),(3,0),(0,3)$, but it does not hold for $\alpha=(1,2),(2,1)$. Thus, this scheme reproduces quadratic polynomials.

\section{Acknowledgements}

This work is supported partially by UM Research Board 10/05, UMSL Research Award 10/06, China Postdoctoral Science Foundation No. 20090460564 and the President Fund of GUCAS. The authors thank two anonymous referees for their valuable suggestions and comments.

\section{References}

[1] C. Cabrelli, C. Heil, and U. Molter, Accuracy of lattice translates of several multidimensional refinable functions, Journal of Approximation Theory, 95 (1998) 5-52.

[2] E. Catmull and J. Clark, Recursively generated b-spline surfaced on arbitrary topological meshes, Computer-Aided Design, 10 (1978) 350355 . 
[3] C.K. Chui and Q.T. Jiang, Matrix-valued symmetric templates for interpolatory surface subdivisions I. Regular vertices, Applied and Computational Harmonic Analysis, 19 (2005) 303-339.

[4] C.K. Chui and Q.T. Jiang, Matrix-valued subdivision schemes for generating surfaces with extraordinary vertices, Computer Aided Geometric Design, 23 (2006) 419-438.

[5] C. Heil, G. Strang, and V. Strela, Approximation by translates of refinable functions, Numerische Mathematik, 73 (1996) 75-94.

[6] R.Q. Jia and Q.T. Jiang, Approximation power of refinable vectors of functions, in: Wavelet analysis and applications, Ams/Ip Studies in Advanced Mathematics, Vol. 25, Amer. Math. Soc., Providence, RI, 2002, pp. 155-178.

[7] Q.T. Jiang, Multivariate matrix refinable functions with arbitrary matrix dilation, Transactions of the American Mathematical Society, 351 (1999) 2407-2438.

[8] Q.T. Jiang, B.B. Li, and W.W. Zhu, Interpolatory quad/triangle subdivision schemes for surface design, Computer Aided Geometric Design, 26 (2009) 904-922.

[9] A. Levin, Polynomial generation and qusi-interpolation in stationary non-uniform subdivision, Computer Aided Geometric Design, 20 (2003) 41-54.

[10] A. Levin and D. Levin, Analysis of quasi-uniform subdivision, Applied and Computational Harmonic Analysis, 15 (2003) 18-32.

[11] C. Loop, Smooth subdivision surfaces based on triangles, University of Utah, Department of Mathematics, Masters Thesis, 1987.

[12] J. Maillot and J. Stam, A unified subdivision scheme for polygonal modeling, Proceedings of Eurographics 2001, Computer Graphics Forum, 20 (2001) 471-479.

[13] J. Peters and L.-J. Shiue, Combining 4- and 3-direction subdivision, ACM Transactions on Graphics, 23 (2004) 980-1003. 
[14] G. Plonka, Approximation order provided by refinable function vectors, Constructive Approximation, 13 (1997) 221-244.

[15] S. Schaefer and J. Warren, On $C^{2}$ triangle/quad subdivision, ACM Transactions on Graphics, 24 (2005) 28-36.

[16] J. Stam and C. Loop, Quad/triangle subdivision, Computer Graphics Froum, 22 (2003) 1-7. 